\title{
Endogenous Brain-Derived Neurotrophic Factor in the Nucleus Tractus Solitarius Tonically Regulates Synaptic and Autonomic Function
}

\author{
Catharine G. Clark, ${ }^{1,2}$ Eileen M. Hasser, ${ }^{1,2,3}$ Diana L. Kunze, ${ }^{4,5}$ David M. Katz, ${ }^{5 *}$ and David D. Kline ${ }^{1,2 *}$ \\ ${ }^{1}$ Dalton Cardiovascular Research Center, Departments of ${ }^{2}$ Biomedical Sciences, and ${ }^{3}$ Medical Pharmacology and Physiology, University of Missouri, \\ Columbia, Missouri 65211, ${ }^{4}$ Rammelkamp Center, MetroHealth Systems, Cleveland, Ohio 44109, and ${ }^{5}$ Department of Neurosciences, Case Western Reserve \\ University, Cleveland, Ohio 44106
}

Brain-derived neurotrophic factor (BDNF) and its receptor, TrkB, are highly expressed in the nucleus tractus solitarius (nTS), the principal target of cardiovascular primary afferent input to the brainstem. However, little is known about the role of BDNF signaling in nTS in cardiovascular homeostasis. We examined whether BDNF in nTS modulates cardiovascular function in vivo and regulates synaptic and/or neuronal activity in isolated brainstem slices. Microinjection of BDNF into the rat medial nTS (mnTS), a region critical for baroreflex control of sympathetic outflow, produced dose-dependent increases in mean arterial pressure (MAP), heart rate (HR), and lumbar sympathetic nerve activity (LSNA) that were blocked by the tyrosine kinase inhibitor K252a. In contrast, immunoneutralization of endogenous BDNF (anti-BDNF), or microinjection of K252a alone, decreased MAP, HR, and LSNA. The effects of anti-BDNF were abolished by blockade of ionotropic glutamate receptors, indicating a role for glutamate signaling in the response to BDNF. In vitro, BDNF reduced the amplitude of miniature EPSCs as well as solitary tract (TS) evoked EPSC amplitude and action potential discharge (APD) in second-order nTS neurons. BDNF effects on EPSCs were independent of GABAergic signaling and abolished by AMPA receptor blockade. In contrast, K252a increased spontaneous EPSC frequency and TS evoked EPSC amplitude. BDNF also attenuated APD evoked by injection of depolarizing current into second-order neurons, indicating reduced intrinsic neuronal excitability. Our data demonstrate that BDNF signaling in mnTS plays a tonic role in regulating cardiovascular function, likely via modulation of primary afferent glutamatergic excitatory transmission and neural activity.

\section{Introduction}

The brainstem nucleus tractus solitarius (nTS) is the primary central target of peripheral visceral sensory neurons, including baroreceptor and chemoreceptor afferents, and thus plays a critical role in regulating cardiorespiratory homeostasis. Moreover, nTS contains the initial synaptic relay at which blood pressurerelated sensory information is subject to neuromodulatory influences that affect reflex control of cardiovascular parameters including arterial blood pressure, heart rate and sympathetic nerve activity. Brain-derived neurotrophic factor (BDNF), a potent modulator of synaptic transmission, is expressed by neurons involved in cardiovascular regulation at multiple levels of the

Received Feb. 10, 2011; revised July 7, 2011; accepted July 12, 2011.

Author contributions: C.G.C., E.M.H., D.L.K., D.M.K., and D.D.K. designed research; C.G.C., E.M.H., and D.D.K. performed research; C.G.C., E.M.H., and D.D.K. analyzed data; C.G.C., E.M.H., D.L.K., D.M.K., and D.D.K. wrote the paper.

This research was supported by NIH Grants NS-057398 (D.M.K.) and 5T32AR048523-05 (Dr. Ronald Terjung), HL-085108 (D.D.K.), and HL-55306 (E.M.H.) and American Heart Association Grants Midwest Affiliate 09PRE2260878 (C.G.C.). We acknowledge Sarah Friskey for her expert technical assistance.

*D.M.K. and D.D.K. are co-senior authors.

Correspondence should be addressed to Dr. David D. Kline, Department of Biomedical Sciences, University of Missouri, Dalton Cardiovascular Research Center, 134 Research Park Drive, Columbia, M0, 65211. E-mail: klinedd@missouri.edu.

DOI:10.1523/JNEUROSCI.0746-11.2011

Copyright $\odot 2011$ the authors $\quad 0270-6474 / 11 / 3112318-12 \$ 15.00 / 0$ neuraxis, including baroreceptors and chemoafferents in the nodose and petrosal sensory ganglia (Brady et al., 1999; Martin et al., 2008) as well as nTS neurons (Conner et al., 1997). In addition, nodose-petrosal ganglion (NPG) neurons release BDNF in an activity-dependent fashion (Balkowiec and Katz, 2000), including at discharge frequencies consistent with baroreceptor activation (Andresen and Kunze, 1994). However, relatively little is known about the role of BDNF in central regulation of cardiovascular homeostasis in general, and in nTS in particular.

Glutamate is the major excitatory transmitter of NPG afferent terminals (Gordon and Leone, 1989; Ohta and Talman, 1994; Aylwin et al., 1998) and BDNF has been shown to attenuate glutamatergic AMPA currents in isolated nTS neurons (Balkowiec et al., 2000). Moreover, BDNF levels in NPG neurons and nTS are severely depleted in mouse models of Rett syndrome (RTT) (Wang et al., 2006b; Ogier et al., 2007; Kline et al., 2010), a developmental disorder characterized in part by dysregulation of cardiorespiratory homeostasis, and exogenous BDNF can rescue synaptic dysfunction in nTS slice preparations in Mecp2 mutants (Kline et al., 2010). Together, these data support a role for BDNF in modulating excitatory synaptic transmission between primary afferents and second-order nTS neurons involved in cardiovascular autonomic control.

To test the hypothesis that BDNF contributes to regulation of cardiovascular homeostasis in vivo by modulating neurotrans- 
mission in the nTS, we microinjected BDNF directly into the medial nTS (mnTS) of anesthetized rats while simultaneously monitoring arterial blood pressure (ABP), heart rate (HR) and lumbar sympathetic nerve activity (LSNA). To determine whether endogenous BDNF acts tonically in mnTS, we microinjected a BDNF scavenging antibody (anti-BDNF) or a BDNF receptor (TrkB) antagonist, K252a. We found that in vivo administration of BDNF increased mean arterial pressure (MAP), HR, and LSNA, whereas scavenging BDNF or inhibition of TrkB signaling decreased MAP, HR, and LSNA. The tonic actions of BDNF in vivo were abolished by ionotropic glutamate receptor blockade. To investigate underlying mechanisms, we used the patch-clamp technique in a brainstem slice preparation to record from mnTS neurons receiving monosynaptic primary afferent input in the absence and presence of BDNF. In vitro application of BDNF decreased spontaneous EPSC frequency and amplitude, solitary tract (TS)-afferent evoked EPSC amplitude and action potential discharge (APD), and miniature EPSC amplitude. BDNF also reduced APD to current injection into nTS neurons. Together, our data indicate that BDNF modulates synaptic transmission and neuronal activity in mnTS and contributes tonically to the regulation of cardiovascular homeostasis.

\section{Materials and Methods Ethical approval}

All procedures were approved by the Animal Care and Use Committee of the University of Missouri. Adult male Sprague Dawley rats (Harlan) were maintained on a $12 \mathrm{~h}$ light/dark cycle with food and water available ad libitum. All rats were given at least $7 \mathrm{~d}$ to acclimate to the surroundings before any experimental procedure.

\section{BDNF immunohistochemistry}

Deeply anesthetized rats were transcardially perfused with heparinized, oxygenated tissue culture medium (300 ml, DMEM (DMEM-F12, Sigma-Aldrich), followed by $500 \mathrm{ml}$ of $4 \%$ paraformaldehyde (SigmaAldrich). Brains were removed and postfixed overnight. Sections (coronal or horizontal) were generated on a vibratome $(30 \mu \mathrm{m}$, Leica VT $1000 \mathrm{~S}$ ) and processed immunohistochemically for BDNF.

Sections were rinsed for $30 \mathrm{~min}$ in $0.1 \mathrm{M}$ PBS, followed by heat-induced epitope retrieval in a decloaking chamber (Biocare Medical). Sections were then rinsed for $30 \mathrm{~min}$ in $0.1 \mathrm{M}$ PBS followed by a blocking solution [10\% normal donkey serum (NDS) Jackson ImmunoResearch] in PBSTriton X-100 0.3\% v/v (PBST) for $30 \mathrm{~min}$ ). Sections were incubated overnight in chicken anti-BDNF (1:25) (Martin et al., 2008) with $1 \%$ NDS in $0.1 \mathrm{M}$ PBST. After primary incubation, sections were rinsed in 0.1 M PBS for 30 min then incubated in donkey-anti-chicken IgG conjugated to CY3 (1:200), in 3\% NDS in $0.1 \mathrm{M}$ PBS. Sections were rinsed in $0.1 \mathrm{M}$ PBS for 30 min, mounted onto gelatin-coated slides, dried, coverslipped with Prolong Gold (Invitrogen) and sealed with clear nail polish. Control sections were treated as described above, except that the primary antibody was omitted. In addition, we verified the specificity of the BDNF antibody by preabsorbing the antibody with $\operatorname{BDNF}(1: 10 ; 16 \mathrm{~h})$ before using it in the immunohistochemical protocol. Sections were viewed and images captured on an Olympus microscope (BX51) equipped with a monochrome digital camera (ORCA-AG, Hamamatsu) and a filter set for $\mathrm{Cy} 3$ [excitation, $\lambda 550 \mathrm{~nm}$; emission, $\lambda 570 \mathrm{~nm}$ ]. Acquisition of images was performed using the software package Neurolucida (version 7.5, MicroBrightField). Images were imported into ImageJ (version 1.43, $\mathrm{NIH}$ ) and adjusted for brightness and contrast.

\section{In vivo studies}

Surgical procedures

Male rats ( $8-10$ weeks of age), were anesthetized with isoflurane [Forane, Baxter (5\% in $100 \% \mathrm{O}_{2}, 2 \mathrm{~L} / \mathrm{min}$, maintenance $\left.\left.2-2.5 \%\right)\right]$. Catheters (PE10 fused to PE50) were inserted into the aorta via the femoral artery for recording arterial pressure and into the vena cava via the femoral vein to facilitate administration of drugs. Arterial pressure was recorded using a pressure transducer connected to a DC amplifier (ADInstruments), and MAP and HR were determined using a PowerLab Data Acquisition System (ADInstruments). Rats underwent a tracheotomy and were ventilated mechanically with room air supplemented with $100 \% \mathrm{O}_{2}$. To record LSNA, a midline laparotomy was performed, and the lumbar chain was isolated caudal to the left renal vein. Electrodes used for recording LSNA consisted of two Teflon-insulated silver wires $(0.005$ inch diameter, 36 gauge; Medwire) threaded through SILASTIC tubing ( 0.025 inch inside diameter) and formed into a hook. The electrodes were placed around the isolated sympathetic chain and covered with polyvinylsiloxane gel (President, Coltène/Whaledent Inc), which was allowed to harden before closure. LSNA was amplified 1000 times using a Grass preamplifier (P511) and filtered $(30 \mathrm{~Hz}-3 \mathrm{kHz})$. Raw sympathetic nerve activity underwent rectification and integration using a root mean square (rms) converter (time constant: $28 \mathrm{~ms}$ ) and was averaged electronically. Background noise was determined from the residual LSNA signal following euthanasia. The magnitude of the recorded nerve activity minus background noise was defined as LSNA. The level of LSNA under baseline conditions was designated as $100 \%$.

\section{Brainstem microinjections}

Rats were placed in a stereotaxic device (Kopf). The dorsal surface of the medulla was visualized through an occipital craniotomy. Briefly, the dorsal neck muscles were separated to expose the atlanto-occipital membrane, the membrane removed and calamus scriptorius, defined as the caudal pole of the area postrema, was visualized. Once the dorsal medulla was exposed, rats were gradually removed from Isoflurane while a series of Inactin injections $(0.05 \mathrm{ml}, 100 \mathrm{mg} / \mathrm{ml}$, i.v. for a total dose of 100 $\mathrm{mg} / \mathrm{kg}$ over $\sim 30 \mathrm{~min}$ ) was administered. A triple barrel glass micropipette (outside tip $\sim 40 \mu \mathrm{m}$ ) containing agents appropriate for the specific experimental protocol was inserted into the mnTS under visual guidance using a surgical microscope. Microinjections were made using a custom built pressure injection system. To directly measure the volumes microinjected, movement of fluid in the pipette barrel was visualized through a $150 \times$ microscope (Rolyn Optics) with a fine reticule. Target stereotaxic coordinates for the nTS were $0.5 \mathrm{~mm}$ rostral and $0.5 \mathrm{~mm}$ lateral to calamus scriptorius and $0.5 \mathrm{~mm}$ ventral from the dorsal surface of the medulla. All agonists were microinjected unilaterally. To determine the overall tonic role of endogenous BDNF, antagonists were microinjected bilaterally. In experimental protocols which required bilateral microinjections, the pipette was withdrawn from the initial injection site and reinserted on the contralateral side of the brain within $\sim 1 \mathrm{~min}$.

\section{Protocols}

Functional and histological identification of the medial nTS. The nTS was identified by a depressor and sympathoinhibitory response (at least 25 $\mathrm{mmHg}$ and $50 \%$ decrease in LSNA, respectively) to microinjection of L-glutamate $(10 \mathrm{~mm} ; 30 \mathrm{nl})$. Injection sites were further verified by standard histological evaluation. At the end of experiments, deeply anesthetized rats were transcardially perfused and brains prepared for immunohistochemistry as described above. A donkey-anti-Chicken-IgG conjugated to Cy3 (1:200) was used to visualize anti-BDNF. Locations of injection sites were estimated by comparison to a rat brain atlas (Paxinos and Watson, 1998).

Effect of exogenous BDNF in the nTS on cardiovascular parameters. Increasing doses of BDNF (50, 100, 200 and $500 \mathrm{ng} / \mathrm{ml} ; 30 \mathrm{nl}$ ) were unilaterally microinjected into the nTS as described above and cardiovascular parameters (MAP and HR) and LSNA were monitored for 60 min following the injection. Control experiments consisted of microinjection of $30 \mathrm{nl}$ of artificial CSF ( $\mathrm{aCSF}$ ). To eliminate potential confounding effects of BDNF on subsequent microinjections, each animal received only one dose of BDNF and the order of experiments was varied to produce a balanced design.

An additional set of experiments was performed to verify that the effects of BDNF on MAP, HR, and LSNA were mediated by its receptor, TrkB. The tyrosine kinase inhibitor K252a (200 nM; $90 \mathrm{nl}$ ) was bilaterally microinjected into the nTS, followed within 5 min by bilateral microinjections of BDNF (200 ng/ml; $30 \mathrm{nl})$. Cardiovascular parameters (MAP, HR) and LSNA were measured for $60 \mathrm{~min}$. 
Tonic role of endogenous BDNF in the nTS in cardiovascular function. To assess the overall tonic influence of BDNF in the nTS, cardiovascular parameters (MAP and HR) and LSNA were evaluated for 60 min under control conditions and following bilateral microinjection of anti-BDNF $(10 \mu \mathrm{g} / \mathrm{ml} ; 90 \mathrm{nl})$ or K252a (200 nM; $90 \mathrm{nl})$. Anti-BDNF, a BDNF scavenging antibody, selectively immnunoneutralizes endogenous BDNF (Balkowiec and Katz, 2000; Wang et al., 2006a,b) and has been used in similar in vivo preparations (Alonso et al., 2002; Husson et al., 2005; Sanchez et al., 2006; Faraguna et al., 2008). In control experiments, bilateral microinjections of a structurally similar control antibody to anti-BDNF (chicken IgY; $10 \mu \mathrm{g} / \mathrm{ml} ; 90 \mathrm{nl}$ ) or vehicle (aCSF) were also administered.

To confirm the specificity of anti-BDNF's cardiovascular effects, a separate set of experiments was performed $(n=4)$ in which BDNF (500 $\mathrm{ng} / \mathrm{ml})$ was preabsorbed with anti-BDNF $(10 \mu \mathrm{g} / \mathrm{ml})$. Briefly, BDNF and anti-BDNF were combined $24 \mathrm{~h}$ before the experiment and incubated at $4^{\circ} \mathrm{C}$ on a laboratory shaker. The BDNF-anti-BDNF preabsorption mixture $(90 \mathrm{nl})$ was microinjected bilaterally and cardiovascular parameters (MAP and HR) were monitored for $60 \mathrm{~min}$.

$B D N F$ and ionotropic glutamate receptors. To test the effects of ionotropic glutamate receptor blockade on the tonic action of endogenous $\mathrm{BDNF}$, the glutamate receptor antagonist kynurenic acid (KYN; $40 \mathrm{~mm}$; $60 \mathrm{nl}$ ) was microinjected bilaterally into the nTS. Kynurenic acid was used to block both non-NMDA- and NMDA-type ionotropic glutamate receptors because both types of receptors have been shown to play a role in cardiovascular regulation in the nTS in vivo (Ohta and Talman, 1994). Following administration of KYN, anti-BDNF was subsequently bilaterally microinjected, as described above. Cardiovascular parameters (MAP and HR) and LSNA were monitored for an additional $60 \mathrm{~min}$.

\section{In vitro studies}

\section{Brainstem $n T S$ slice preparation}

In a separate group of 5-8 week-old male rats, brainstem slices containing the nTS were prepared. Animals were anesthetized with Isoflurane and decapitated. The brainstem was rapidly removed and placed in icecold aCSF containing the following (in mM): $124 \mathrm{NaCl}, 25 \mathrm{NaHCO}_{3}, 3.0$ $\mathrm{KCl}, 1 \mathrm{MgSO}_{4}-7 \mathrm{H}_{2} \mathrm{O}, 1.5 \mathrm{NaH}_{2} \mathrm{PO}_{4}, 10$ glucose, and $1.5 \mathrm{CaCl}_{2}$, saturated with $95 \% \mathrm{O}_{2}-5 \% \mathrm{CO}_{2}(\mathrm{pH} 7.4,300 \mathrm{mOsm})$. Horizontal slices ( 280 $\mu \mathrm{m}$ ) were cut using a vibratome (Leica VT 1000S).Tissue sections were placed in a superfusion chamber, secured with nylon mesh and superfused with aCSF at a flow rate of $4 \mathrm{ml} / \mathrm{min}\left(34^{\circ} \mathrm{C}\right)$. Neurons were visualized using an Olympus microscope equipped with differential interface contrast and an infrared-sensitive camera. The recording pipette was guided using a piezoelectric micromanipulator (PCS-6000, Burleigh). Recording electrodes contained the following (in $\mathrm{mM}$ ): $10 \mathrm{NaCl}, 130 \mathrm{~K}^{+}$ gluconate, $11 \mathrm{EGTA}, 1 \mathrm{CaCl}_{2}, 10 \mathrm{HEPES}, 1 \mathrm{MgCl}_{2}, 2 \mathrm{MgATP}$, and 0.2 NaGTP, pH 7.3, 295-300 mOsm. Data were recorded using a Multiclamp $700 \mathrm{~B}$ amplifier, filtered at $2 \mathrm{kHz}$ and sampled at $10 \mathrm{kHz}$ using pClamp programs (Molecular Devices). All recordings were made in the medial nTS. Cells were rejected if the resting potential was more positive than $-45 \mathrm{mV}$ under current-clamp mode on initial membrane rupture. BDNF and K252a were dissolved in aCSF and bath applied.

\section{Protocols}

Effects of BDNF and blockade of TrkB on spontaneous EPSCs. Spontaneous EPSCs (sEPSCs) constitute network activity within the slice and were recorded in the absence of external stimulation. Comparison of sEPSC frequency and amplitude in the presence of BDNF (100 ng/ml, $30 \mathrm{~min})$ or K252a (200 nM, $30 \mathrm{~min}$ ) was made to control (aCSF) in the identical neuron. Cells were voltage-clamped at $-60 \mathrm{mV}$.

Effects of BDNF and blockade of TrkB on solitary tract evoked EPSCs and action potential discharge. Synaptic currents were evoked using a concentric bipolar stimulating electrode (F. Haer) placed on TS. Stimulation intensity was increased until an EPSC was evoked and final TS intensity was set at $1.5 \times$ threshold (range $12-900 \mu \mathrm{A}$ ). The TS was stimulated at $0.5 \mathrm{~Hz}(0.1 \mathrm{~ms}$ duration) for 20 episodes with an isolated programmable stimulator (AMPI). Baseline TS-EPSCs were recorded for $10 \mathrm{~min}$. Any TS shock that failed to produce an identifiable EPSC was considered a synaptic failure. TS-EPSCs were subsequently recorded in the same neu- rons in the presence of BDNF ( $100 \mathrm{ng} / \mathrm{ml}), \mathrm{K} 252 \mathrm{a}(200 \mathrm{nM})$ or aCSF. Cells were voltage-clamped at $-60 \mathrm{mV}$. To examine the effects of BDNF or K252a on the ratio of two consecutive EPSCs (paired pulse ratio) in an individual neuron, two TS stimuli were delivered at an interval of $50 \mathrm{~ms}$ and the ratio of the amplitudes of EPSC2/EPSC1 was calculated in the presence of vehicle and subsequently during superfusion with BDNF (100 ng/ml) or K252a (200 nm).

In some experiments, the effect of BDNF $(100 \mathrm{ng} / \mathrm{ml})$ on evoked EPSCs was recorded in the presence of the $\mathrm{GABA}_{\mathrm{A}}$ antagonist gabazine (SR $95531 \mathrm{HBr}, 25 \mu \mathrm{M}$ ) to eliminate potential effects of BDNF on GABAergic mechanisms. Subsequently, the non-NMDA glutamate receptor antagonist 6-cyano-7-nitroquinoxaline-2,3-dione (CNQX, $10 \mu \mathrm{M}$ ) was added to the perfusate to verify that EPSCs were glutamatergic (Andresen and Yang, 1990).

In a separate series of experiments, APD was induced by TS stimulation $(20 \mathrm{~Hz} ; 20$ events, 10 sweeps) in current-clamp mode. Comparisons of the number of TS-evoked APs in the presence of BDNF $(100 \mathrm{ng} / \mathrm{ml})$ were made to control (aCSF).

Effects of BDNF on miniature EPSCs. Miniature currents were recorded in nTS neurons in the absence and presence of BDNF $(100 \mathrm{ng} / \mathrm{ml})$ to examine presynaptic versus postsynaptic effects of BDNF on synaptic transmission. In these experiments, Tetrodotoxin (TTX, $1 \mu \mathrm{M}$ ) and gabazine $(25 \mu \mathrm{M})$ were included in the bath. The lack of evoked APD to depolarizing current steps verified sodium channel blockade and that miniature events were not attributable to action potential-driven spontaneous network activity. Following recording of baseline miniature EPSCs (mEPSCs), BDNF was added to the perfusate and mEPSCs monitored. CNQX was subsequently added to the perfusate to verify that mEPSCs were glutamatergic (Andresen and Yang, 1990).

Effects of BDNF on current-evoked action potential discharge. APD was recorded in current-clamp mode at resting membrane potential. Action potentials were induced by depolarizing current injection $(10-100 \mathrm{pA}$ at $10 \mathrm{pA}, 500 \mathrm{~ms}$ steps). Subsequently, BDNF $(100 \mathrm{ng} / \mathrm{ml})$ was superfused and while recording from the same neuron, effects on action potential discharge evaluated.

\section{Drugs and antibodies}

Inactin, L-glutamate, kynurenic acid and DMEM-F12 were obtained from Sigma-Aldrich. BDNF was obtained from Cell Sciences, Inc., antiBDNF was obtained from Promega and K252a was obtained from Calbiochem. Gabazine (SR 95531 hydrobromide), CNQX and TTX were obtained from Tocris Bioscience. Donkey anti-chicken conjugated to Cy3 was obtained from Jackson ImmunoResearch. K252a was initially dissolved in DMSO at $2 \mathrm{~mm}$. The final concentration of DMSO in the working solutions was $0.01 \%$ and has been shown to have no effect on neuronal function (Kang and Schuman, 1995). Kynurenic acid was first dissolved in $1 \mathrm{~N} \mathrm{NaOH}$ before being diluted in aCSF. anti-BDNF was dissolved in $1 \%$ bovine serum albumin (BSA). All drugs were $\mathrm{pH}$ adjusted to 7.3-7.5 using sodium hydroxide or hydrochloric acid and filtered before microinjection.

\section{Data analysis}

Data for in vivo experiments were acquired using PowerLab (ADInstruments) and analyzed with SigmaStat. Dose-related effects of BDNF were compared by one-way ANOVA. Effects relative to baseline for a given intervention were evaluated by paired $t$ test. Data comparing peak changes in MAP, HR, or LSNA in response to these interventions were analyzed by one-way ANOVA. In all animals, baseline LSNA was defined as $100 \%$ as described above. Any drug-related changes $(\Delta)$ due to experimental interventions were calculated as a percentage of this baseline ( $\%$ of control). Electrophysiological data were analyzed via Clampfit (Molecular Devices) and Synaptosoft Mini-Analysis Program software. EPSC latency was determined as the time between the onset of the stimulus artifact and the beginning of the synaptic current. Jitter was calculated as the SD of latency for each cell (Doyle and Andresen, 2001). Jitter values $<$ $200 \mu$ s were considered to indicate monosynaptic connections. Data from brain slice experiments are presented as the average of 20 current sweeps. Any TS shock that failed to produce an identifiable EPSC was 

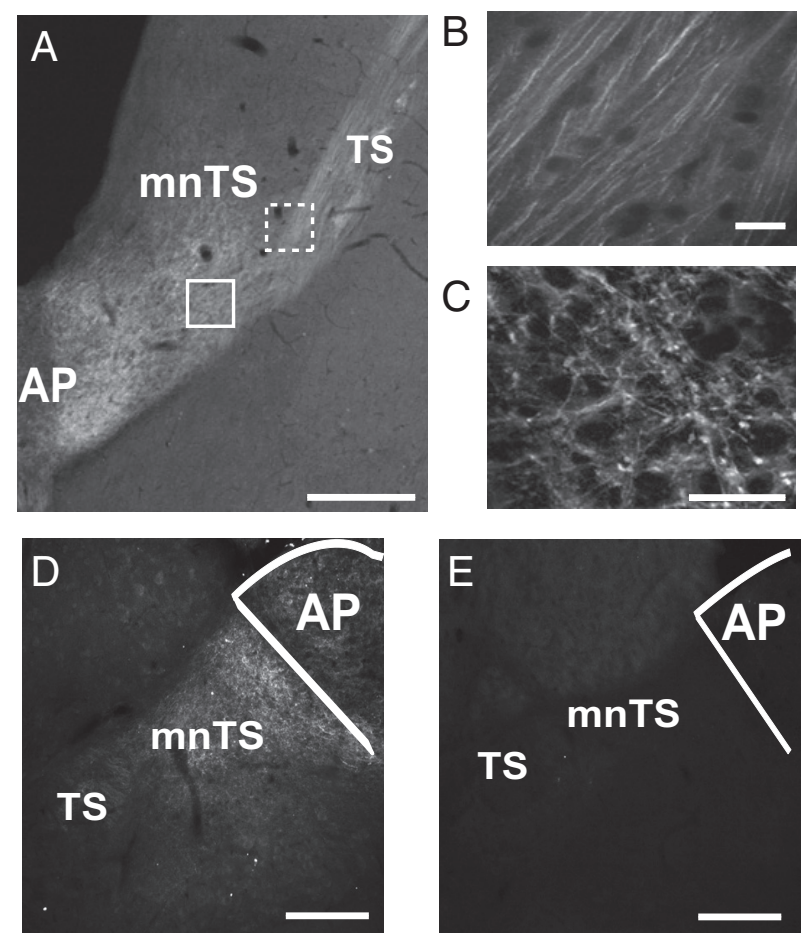

$\mathrm{F}$
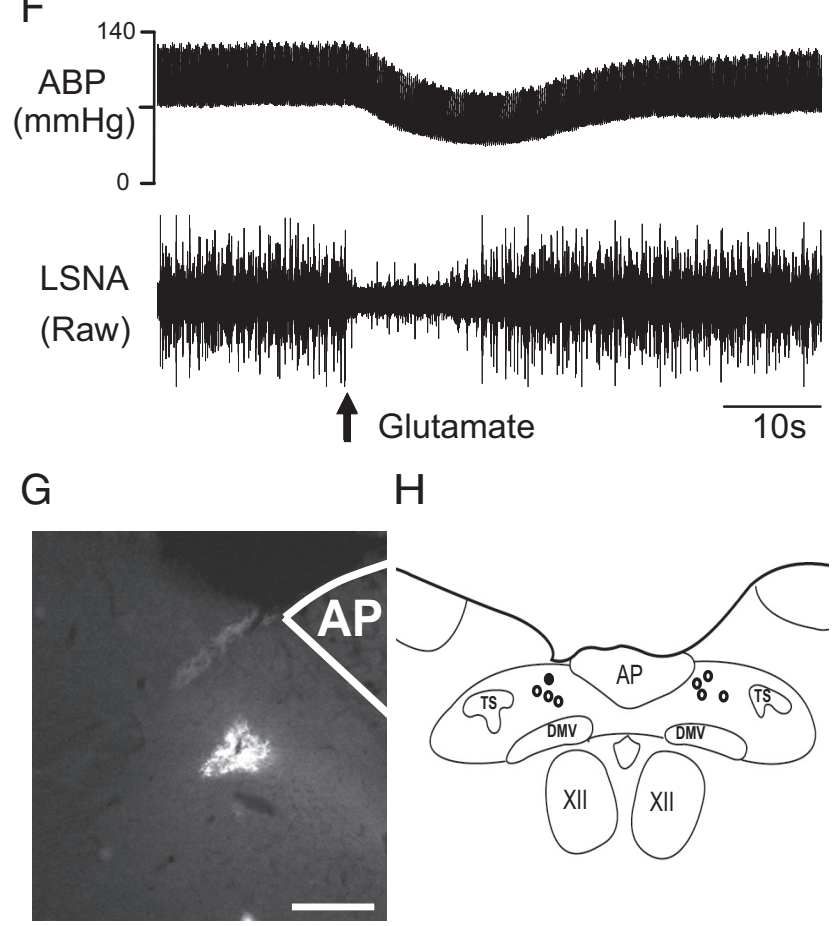

Figure 1. nTS microinjection sites correspond to regions of BDNF expression. $\boldsymbol{A}, \mathrm{BDNF}$ is highly expressed in the medial nTS and solitary tract (horizontal slice). Scale bar, $500 \mu \mathrm{m} . \boldsymbol{B}, \boldsymbol{C}$, High magnification of BDNF immunoreactivity localized to afferent fibers in the solitary tract ( $\boldsymbol{B}$; region in dashed box in $\boldsymbol{A}$; scale bar, $25 \mu \mathrm{m}$ ) and surrounding neuropil ( $\boldsymbol{C}$; region in solid box in $\boldsymbol{A}$; scale bar, $50 \mu \mathrm{m})$. D, BDNF immunoreactivity in a coronal nTS section illustrating BDNF labeling in the medial nTS region. Scale bar, $250 \mu \mathrm{m}$. $\boldsymbol{E}$, Immunohistochemical control in which BDNF immunolabeling was eliminated by preabsorption of the antibody with recombinant BDNF. $\boldsymbol{F}$, Representative recording from one animal demonstrating the arterial blood pressure $(A B P)$ and LSNA responses to unilateral microinjection of glutamate $(30 \mathrm{nl} ; 10 \mathrm{~mm})$. The medial $n T S$ region was identified by the presence of a depressor $(>25 \mathrm{mmHg}$ ) and sympathoinhibitory ( $>50 \%$ reduction) response to glutamate. $G$, Representative example verifying the location of anti-BDNF microinjection in the medial nTS region in one animal (represented by closed circle in G). Anti-BDNF was visualized with an anti-chicken antibody conjugated to Cy3. Note that the counted as a synaptic failure. Failure rates were calculated as a percentage of total TS shocks delivered for that cell. Holding currents were averaged from a $2 \mathrm{~ms}$ period (without synaptic activity), obtained during the 0.5 $\mathrm{Hz}$ stimulation protocol. EPSC decay time and tau $(\tau)$ were determined by fitting current decay from 90 to $10 \%$ of its peak value. Data evaluating effects of BDNF or K252a on evoked EPSC amplitude, paired-pulse ratio, sEPSCs, mEPSCs, EPSC tau and decay time, APD, input resistance, failure rate and holding currents were analyzed by paired $t$ test. Spontaneous and miniature EPSC frequency and amplitude distributions were compared using Kolmogorov-Smirnov test. Action potential properties were determined from the first current-induced action potential. Statistical analysis was performed with SigmaStat or Mini Analysis. For all analyses, $p<0.05$ was considered statistically significant. Data are expressed as means $\pm \mathrm{SE}$.

\section{Results}

\section{BDNF expression in the rat mnTS}

To define the role of BDNF signaling in nTS in cardiovascular function, we focused on the mnTS, a region that receives, integrates and modulates sensory input from peripheral baroreceptors and is critical for baroreflex control of the sympathetic nervous system. Initial experiments examined the distribution of BDNF protein within mnTS, as this has not previously been described in detail in the rat. Strong immunoreactivity for BDNF protein was observed in fibers in the TS, consistent with expression in primary afferent fibers, and in the neuropil of mnTS, surrounding unstained neuronal cell bodies (Fig. $1 A-D$ ). In control experiments in which primary antibody was withheld (data not shown) or in which the BDNF antibody was preabsorbed with $\operatorname{BDNF}(1: 10$; Fig. $1 E)$, no immunoreactivity was seen.

\section{BDNF signaling in the mnTS tonically modulates cardiovascular homeostasis}

Functional and histological identification of the mnTS

In all microinjection experiments, the location of the pipette in the mnTS was confirmed functionally by depressor and sympathoinhibitory responses to microinjection of glutamate $(10 \mathrm{mM}$; $30 \mathrm{nl}, n=45)$. Figure $1 F$ illustrates the reduction in arterial blood pressure (ABP) and LSNA in response to microinjection of glutamate in the mnTS from one representative animal. Quantitatively, glutamate significantly decreased $\mathrm{MAP}(-31 \pm 1 \mathrm{mmHg})$, HR $[-14 \pm 1$ beats per minute (bpm) $]$, and LSNA $(-64 \pm 2 \%)$, consistent with activation of neurons involved in baroreflex function. Histological analysis of the injection sites verified that pipette placement was within the mnTS (Fig. 1G,H).

\section{$B D N F$ induces a pressor response}

Cardiovascular parameters were measured in all in vivo microinjection experiments. Baseline MAP and $\mathrm{HR}(n=45)$ were $98 \pm 1$ $\mathrm{mmHg}$ and $324 \pm 8 \mathrm{bpm}$, respectively, and baseline levels of LSNA were defined as $100 \%$.

Initial studies determined the role of BDNF in the mnTS on cardiovascular control by monitoring changes in MAP, HR, and LSNA in response to unilateral microinjection of BDNF (50-500 $\mathrm{ng} / \mathrm{ml} ; 30 \mathrm{nl}$ ) or vehicle ( $30 \mathrm{nl}$; aCSF) into the medial $\mathrm{nTS}$. Figure $2 A$ illustrates representative results from one animal. Unilateral microinjection of BDNF (200 ng/ml; $30 \mathrm{nl}$ ) increased MAP, HR,

\section{$\leftarrow$}

injection site corresponds to the region of BDNF expression within the medial $n T S$ region (see $\boldsymbol{D}$ ). Scale bar, $250 \mu \mathrm{m}$. $\boldsymbol{H}$, Summary diagram illustrating the distribution of microinjection sites (circles) within the medial $\mathrm{nTS}$ region in a subset of animals $(n=8)$. Anatomic landmarks are according to Paxinos and Watson (1998). AP, Area postrema, DMV, dorsal motor nucleus of the vagus; XII, hypoglossal nucleus. 
and LSNA $(+8 \mathrm{mmHg},+12 \mathrm{bpm}$ and $+21 \%$ of control, respectively), reaching a peak within 10 min and recovering back to baseline within $1 \mathrm{~h}$. By contrast, as shown in Figure $2 B$, unilateral microinjection of vehicle (aCSF; $30 \mathrm{nl}$ ) did not alter MAP, HR, or LSNA. Mean data (Fig. 3, $n=6-9$ ) indicate that unilateral microinjections of BDNF in the mnTS produced significant dose-dependent increases in MAP, HR, and LSNA. For example, at $200 \mathrm{ng} / \mathrm{ml} \mathrm{BDNF}$ increased MAP $(+8 \pm 1 \mathrm{mmHg}), \mathrm{HR}(+17 \pm 3$ bpm), and LSNA ( $+23 \pm 6 \%$ of baseline). Microinjection of aCSF (open circle, $n=$ 6) had no effect. BDNF responses were significantly greater than baseline and than responses to vehicle (aCSF) injection.

To define the role of tyrosine kinase receptor activity in the actions of BDNF on MAP, HR, and LSNA, BDNF microinjection was preceded by microinjection of the tyrosine kinase inhibitor, K252a. K252a (200 nM; $90 \mathrm{nl}$ ) alone decreased MAP, HR, and LSNA (see below) and completely eliminated the pressor, tachycardic and sympathoexcitatory responses to subsequent microinjection of BDNF $(200 \mathrm{ng} / \mathrm{ml} ; 30 \mathrm{nl})$. In the presence of $\mathrm{K} 252 \mathrm{a}$, the MAP, HR, and LSNA responses to BDNF were $-1 \pm 2 \mathrm{mmHg}$, $4 \pm 10 \mathrm{bpm}$ and $-1 \pm 3 \% \Delta \mathrm{LSNA}$, respectively.

Blockade of endogenous BDNF induces a depressor response

To determine whether BDNF signaling in nTS exerts a tonic effect on baseline cardiovascular parameters, we evaluated MAP, $\mathrm{HR}$, and LSNA responses following inhibition of endogenous BDNF signaling. This was accomplished in two ways - by bilaterally scavenging endogenous BDNF with anti-BDNF (10 $\mu \mathrm{g} / \mathrm{ml} ; 90 \mathrm{nl} ; n=5)$ or by inhibiting $\operatorname{TrkB}$ receptor activation with K252a (200 nM; $90 \mathrm{nl} ; n=4)$. Figure $1 G$ illustrates the restricted spatial distribution of anti-BDNF following microinjection into the mnTS in one animal, as well as the correspondence between the injection site and the distribution of endogenous BDNF protein (Fig. 1D).

Figure $4 A$ is a representative example of the effects of anti$\mathrm{BDNF}$ on $\mathrm{ABP}, \mathrm{HR}$, and LSNA in one animal. In contrast to exogenous BDNF, bilateral microinjection of anti-BDNF in the mnTS decreased ABP, HR, and LSNA ( $\triangle \mathrm{MAP}:-7 \pm 2 \mathrm{mmHg}$, $\Delta$ HR: $-16 \pm 8 \mathrm{bpm}$ and $\Delta \mathrm{LSNA}-33 \pm 7 \%$ of control, respectively, Fig. 5). These effects stabilized at $\sim 10 \mathrm{~min}$ and recovered back to baseline within $1 \mathrm{~h}$. Microinjection of a structurally similar control antibody, IgY $(10 \mathrm{ng} / \mathrm{ml} ; 90 \mathrm{nl} ; n=3)$ had no significant effects ( $\triangle \mathrm{MAP}:+1 \pm 1 \mathrm{mmHg} ; \Delta \mathrm{HR}:+5 \pm 2 \mathrm{bpm}$; and $\Delta$ LSNA: $+5 \pm 3 \%$ of control, respectively, Fig. 5).

In a subset of animals, we examined the specificity of antiBDNF to alter cardiovascular parameters. In these experiments,
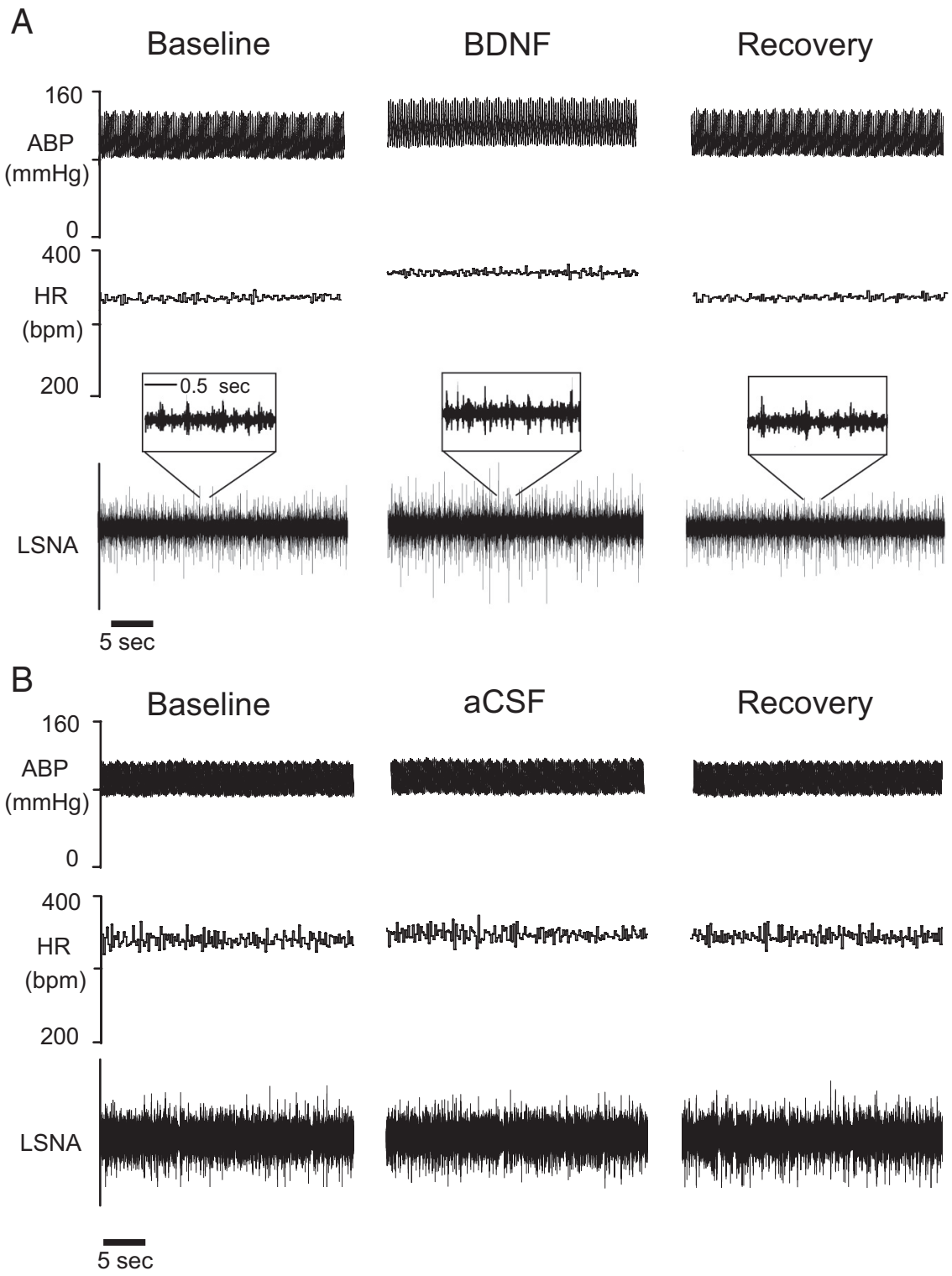

Figure 2. Microinjection of exogenous BDNF into the nTS increases ABP, HR, and LSNA. $A, B$, Representative recordings from nilateral microinjection of BDNF $(200 \mathrm{ng} / \mathrm{ml} ; 30 \mathrm{nl})$ increased ABP, HR, and LSNA. Baseline was taken 1 min before microinjection; DDNF, 10 min following microinjection; Recovery, $1 \mathrm{~h}$ following microinjection of BDNF. Inset in LSNA illustrates expanded raw sympathetic nerve activity from the compressed trace shown below. $\boldsymbol{B}$, In contrast to BDNF, unilateral microinjection of aCSF ( $30 \mathrm{nl})$ into the nTS did not change ABP, HR, or LSNA.

anti-BDNF $(10 \mu \mathrm{g} / \mathrm{ml})$ was preabsorbed with BDNF $(500 \mathrm{ng} / \mathrm{ml})$ and then microinjected ( $90 \mathrm{nl}$ ) bilaterally into the nTS. This preabsorbed mixture had no effect ( $\triangle \mathrm{MAP}:+2 \pm 3 \mathrm{mmHg} ; \Delta \mathrm{HR}:+2 \pm$ $1 \mathrm{bpm})$.

Results similar to those with anti-BDNF were obtained following inhibition of TrkB receptors with the administration of K252a. Bilateral microinjection of K252a significantly decreased MAP, HR, and LSNA $(\Delta ;-7 \pm 2 \mathrm{mmHg},-19 \pm 5 \mathrm{bpm},-25 \pm 7 \%$ of control, respectively, Fig. 5) compared with baseline. The depressor, bradycardic and sympathoinhibitory responses to K252a were not significantly different from those produced by scavenging endogenous BDNF with anti-BDNF (Fig. 5). Microinjection of aCSF had no significant effects and responses were not different from IgY. These data indicate that blockade of endogenous BDNF or TrkB decreases MAP, HR, and SNA to a similar extent. 

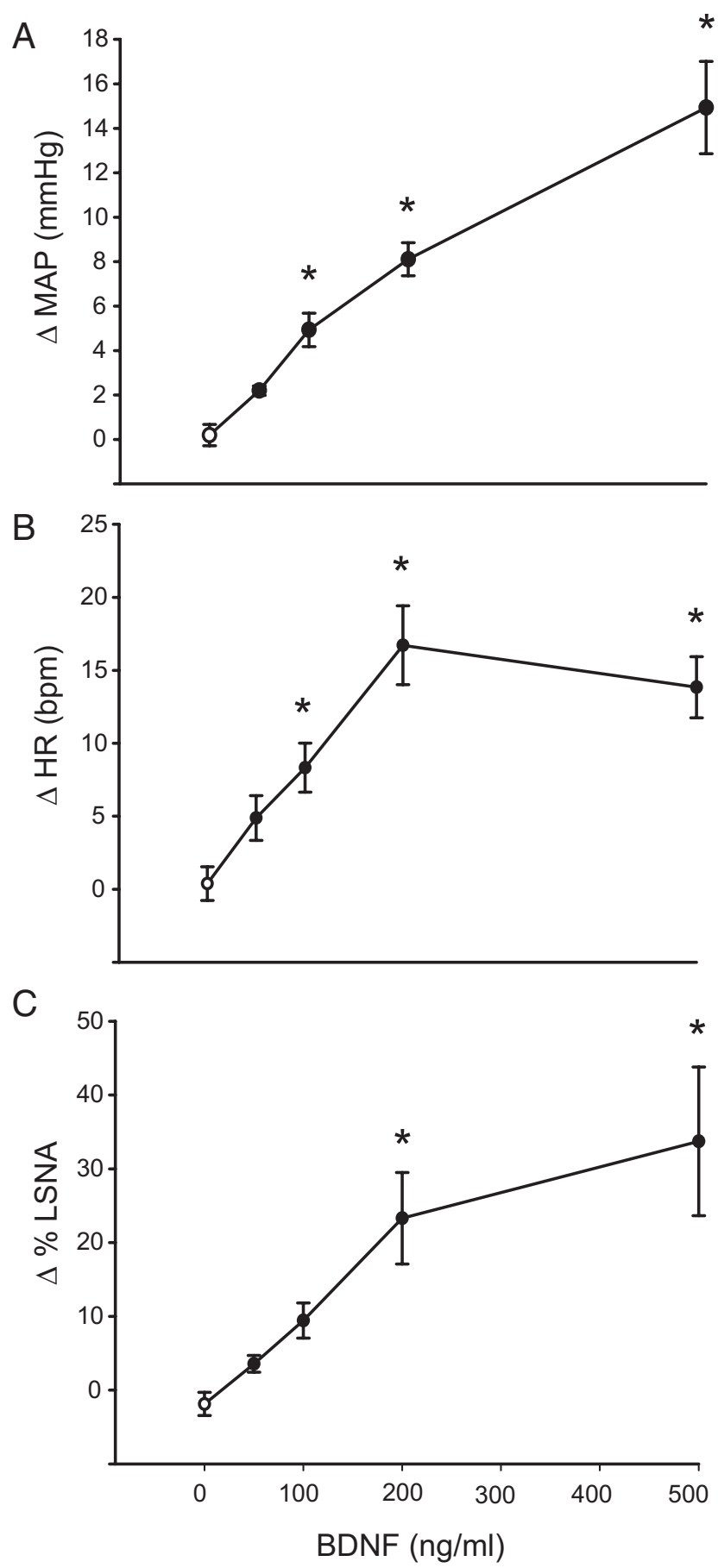

Figure 3. BDNF microinjection in the nTS increases MAP, HR, and LSNA in a dose-related manner. $\boldsymbol{A}-\boldsymbol{C}$, Peak changes in MAP $(\boldsymbol{A}), \mathrm{HR}(\boldsymbol{B})$, and LSNA $(\boldsymbol{C})$ in response to unilateral microinjection (30 nl) of aCSF (open symbols) or BDNF (closed symbols; ${ }^{*} p<0.05$ vs aCSF and baseline; one-way ANOVA). BDNF concentrations were as follows: $50(n=6), 100(n=6), 200$ $(n=9), 500 \mathrm{ng} / \mathrm{ml}(n=9)$ or vehicle (aCSF, $30 \mathrm{nl} ; n=6)$.

Ionotropic glutamate receptor antagonist blocks depressor response to anti-BDNF

Because BDNF modulates glutamatergic neurotransmission and can inhibit AMPA receptor-mediated currents in dissociated nTS neurons in vitro (Balkowiec et al., 2000), we evaluated the role of glutamate receptors in the effects of endogenous BDNF in vivo. KYN (40 mM; $60 \mathrm{nl} ; n=4$ ), an ionotropic glutamate receptor antagonist, was microinjected into the nTS before blockade of endogenous BDNF with anti-BDNF $(10 \mu \mathrm{g} / \mathrm{ml} ; 90 \mathrm{nl})$. As ex- pected, KYN alone increased MAP, HR, and LSNA when microinjected into the nTS ( $\Delta$ MAP: $+15 \pm 3 \mathrm{mmHg} ; \Delta \mathrm{HR}:+20 \pm 3$ bpm; $\triangle$ LSNA: $+25 \pm 4 \%$ of control). In addition, responses to anti-BDNF were eliminated in the presence of KYN. Figure $4 B$ is a representative example from one animal, and group data are shown in Figure 5. During ionotropic glutamate receptor blockade, cardiovascular responses to anti-BDNF were not significantly different from IgY control. These data suggest that under baseline conditions, the cardiovascular and autonomic effects of tonic endogenous BDNF signaling in the nTS are dependent on ionotropic glutamate transmission.

\section{BDNF signaling in the nTS modulates synaptic and neuronal function in vitro}

To define potential mechanisms underlying BDNF-dependent modulation of cardiovascular function in vivo, we analyzed effects of BDNF in vitro using whole-cell patch-clamp recordings from mnTS neurons in an isolated brainstem slice preparation. Specifically, we examined the effects of exogenous BDNF or blockade of TrkB receptors on spontaneous, TS stimulusevoked and miniature EPSCs (mEPSCs) as well as action potential discharge.

$B D N F$ reduces spontaneous (s)EPSCs

To determine the effect of BDNF on nTS network activity, sEPSCs were recorded from identified monosynaptically driven secondorder mnTS cells (see below). Figure $6 \mathrm{~A}$ shows a representative example of sEPSCs recorded under control conditions and in the presence of BDNF in the same neuron. As shown in this example, exogenous BDNF (100 ng/ml, $n=6)$, decreased the frequency and amplitude of sEPSCs compared with control. Cumulative probability plots for interevent interval demonstrated that BDNF produced a significant shift to the right, indicating a decrease in sEPSC frequency (Fig. 6B). Similarly, cumulative probability plots for sEPSC amplitude demonstrated that BDNF produced a significant leftward shift compared with control, indicating a reduction in sEPSC amplitude (Fig. 6C). Quantitatively, BDNF decreased sEPSC frequency from $14.3 \pm 2.0 \mathrm{~Hz}$ to $8.9 \pm 1.7 \mathrm{~Hz}$ (a decrease of $38.6 \pm 7.1 \%, p<0.05$, paired $t$ test) and sEPSC amplitude from $27.7 \pm 2.8 \mathrm{pA}$ to $21.5 \pm 2.9 \mathrm{pA}$ (a decrease of $23.3 \pm 4.3 \%, p<0.05$, paired $t$ test $)$.

BDNF attenuates TS-evoked EPSCs

We next examined the effect of BDNF on TS-evoked EPSCs in these neurons. Only mnTS cells that received direct inputs from the TS (termed monosynaptic cells; Doyle and Andresen, 2001) were analyzed. In defined monosynaptic cells, the mean EPSC latency from shock artifact was $2.4 \pm 0.01 \mathrm{~ms}$ and jitter averaged $151.5 \pm 13.8 \mu \mathrm{s}$. A representative example from a single neuron illustrating the effect of BDNF from baseline on TS-EPSCs is shown in Figure $6 \mathrm{D}$. As shown, bath application of BDNF reduced the amplitude of TS-evoked EPSCs stimulated at $0.5 \mathrm{~Hz}$. Individual and mean data are plotted in Figure 6 E. Evoked EPSC amplitude averaged $481.3 \pm 108.4 \mathrm{pA}(n=6)$ under control (baseline) conditions and was significantly reduced by $21 \pm 6 \%$ to $397.6 \pm 104.7 \mathrm{pA}$ in the presence of BDNF. Compared with vehicle in the same neuron, BDNF had no effect on the pairedpulse ratio between two consecutive EPSCs ( $50 \mathrm{~ms}$ interval; EPSC 2/EPSC 1, control: $0.7 \pm 0.02$ vs BDNF: $0.8 \pm 0.1$ ), and did not alter the likelihood of evoking an EPSC as failure rates were similar (control: $3.1 \pm 0.3 \%$ vs BDNF: $3.1 \pm 0.2 \% ; n=6$ ). BDNF also did not alter TS-EPSC decay time (90-10\%, control: $10.7 \pm 1.3$ ms vs BDNF $11.6 \pm 1.7 \mathrm{~ms})$ or decay tau $\left(\tau_{90-10 \%}\right.$, control: $4.4 \pm$ 
$0.7 \mathrm{~ms}$ vs BDNF $4.3 \pm 0.5 \mathrm{~ms}$ ). A significant reduction in input resistance (control: $584.1 \pm 163.9$ vs BDNF: $243.6 \pm 55.7$ $\mathrm{M} \Omega$ ) accompanied the decrease in EPSC amplitude. In addition, BDNF resulted in a net outward holding current (Control: $-22.3 \pm 6.6$ vs BDNF: $11.1 \pm 9.5 \mathrm{pA})$. Application of vehicle (aCSF, $n=2$ ) did not elicit any changes in evoked EPSC amplitude or input resistance (data not shown).

To examine the possibility that BDNF attenuates synaptic transmission in nTS by altering GABAergic mechanisms, we examined evoked currents in the presence of the $\mathrm{GABA}_{\mathrm{A}}$ receptor antagonist gabazine $(25 \mu \mathrm{M})$ and BDNF (100 ng/ml). In identified second-order cells (latency, $4.27 \pm 0.37 \mathrm{~ms}$; jitter, $121 \pm 7 \mu \mathrm{s})$ gabazine alone had no effect on the amplitude of TS-EPSCs (control: $98.7 \pm 11.9$ vs gabazine: $97.0 \pm 11.1 \mathrm{pA}, n=7)$. Furthermore, in the presence of gabazine, BDNF significantly decreased TS-EPSC amplitude to $80.8 \pm 11.3 \mathrm{pA}(p<0.05, n=7)$, a $19 \pm 4 \%$ reduction compared with gabazine alone. This reduction in TSEPSC amplitude in the presence of gabazine was similar to that without gabazine (above). Subsequent application of the non-NMDA antagonist CNQX significantly and reversibly reduced the amplitude of TS-EPSCs to $11.8 \pm 5.8 \mathrm{pA}$. These data indicate that the reduction in TSEPSC amplitude observed in the presence of BDNF cannot be explained by an increase in GABAergic transmission.

In a separate set of experiments we evaluated the functional significance of BDNF-mediated decreases in synaptic transmission. APD induced by TS stimulation (20 Hz; 20 events; 10 sweeps) was examined in the absence and presence of BDNF (100 ng/ml). Exogenous BDNF significantly attenuated the number of APs evoked by TS stimulation compared with control (control: $39.2 \pm 7.8$ vs BDNF $29.4 \pm$ $9.6 \mathrm{mV}, n=4)$. This effect was independent of membrane potential (Control: $-55.5 \pm$ 8.1 vs BDNF $-54.4 \pm 7.4 \mathrm{mV}, n=4)$.

Tyrosine kinase receptor inhibition augments spontaneous and TS-evoked EPSCS

To examine the possibility that TrkB activation plays a tonic role in regulation of neurotransmission in MnTS, spontaneous and TS-EPSCs were recorded in defined monosynaptic cells (latency, $3.1 \pm 0.4 \mathrm{~ms}$; jitter, $158.2 \pm 33 \mu \mathrm{s}, n=6)$ under baseline conditions and following bath application of K252a (200 nM). As illustrated by the example from an individual neuron shown in Figure $7 A, \mathrm{~K} 252 \mathrm{a}$ increased the frequency and amplitude of sEPSCs compared with baseline. Cumulative probability plots for sEPSC interevent interval demonstrated that K252a produced a signifiresponse to anti-BDNF.
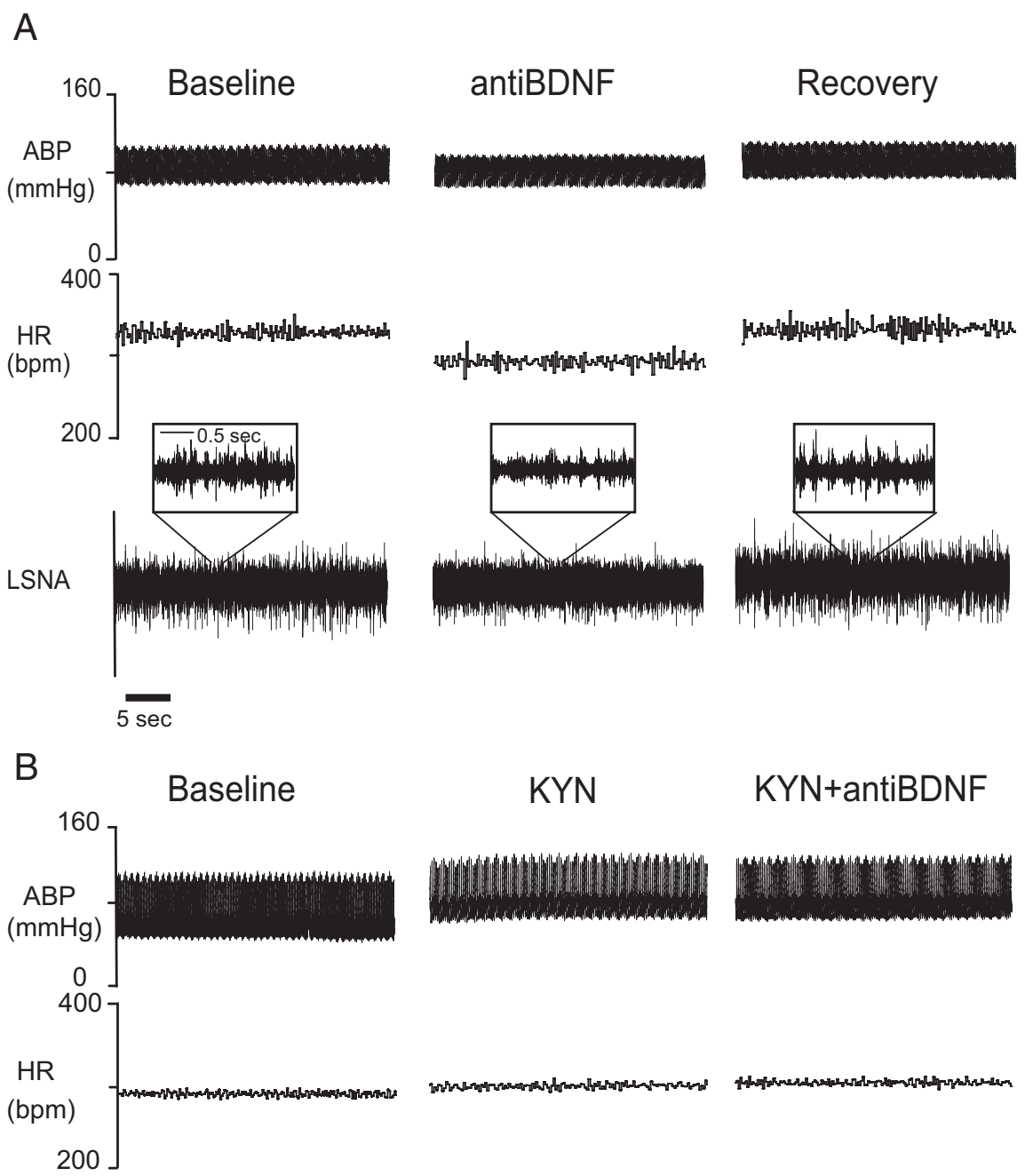

Figure 4. Blockade of endogenous BDNF in the nTS decreases ABP, HR, and LSNA. Representative recordings demonstrating the effect of microinjection of anti-BDNF $(\boldsymbol{A})$ or anti-BDNF after ionotropic glutamate receptor blockade $(\boldsymbol{B})$ in the nTS on ABP, HR, and LSNA. $A$, Example from an individual rat of ABP, HR, and LSNA responses to bilateral $\mathrm{nTS}$ microinjection of anti-BDNF $(10 \mu \mathrm{g} / \mathrm{ml} ; 90$ $\mathrm{nl})$. Note that microinjection of anti-BDNF decreased all three cardiovascular parameters. Baseline was taken 1 min before microinjection; anti-BDNF, 10 min following microinjection; Recovery, $1 \mathrm{~h}$ following microinjection of anti-BDNF. Inset in LSNA, expanded raw sympathetic nerve activity from the compressed trace shown below. $B$, Example from an individual rat of $A B P, H R$, and LSNA responses to a bilateral $\mathrm{nTS}$ microinjection of anti-BDNF $(10 \mu \mathrm{g} / \mathrm{ml} ; 90 \mathrm{nl})$ in the presence of the ionotropic glutamate receptor antagonist KYN (40 mm; $60 \mathrm{nl}$ ) into the nTS. In contrast to anti-BDNF alone; prior microinjection of KYN attenuated the

cant shift to the left, indicating an increase in sEPSC frequency (Fig. 7B). Conversely, K252a produced a significant rightward shift in sEPSC amplitude when compared with baseline, indicating an increase in sEPSC amplitude (Fig. 7C). Quantitatively, K252a increased sEPSC frequency from $5.4 \pm 0.9 \mathrm{~Hz}$ to $10.2 \pm$ $2.9 \mathrm{~Hz}$ (an increase of $82.2 \pm 35.4 \%, p=0.06$, paired $t$ test) and sEPSC amplitude from $33.1 \pm 3.8 \mathrm{pA}$ to $40.3 \pm 3.5 \mathrm{pA}$ (an increase of $24.6 \pm 8.0 \%, p<0.05$, paired $t$ test).

An example of the effect of K252a on TS-evoked ESPCs in a single neuron is shown in Figure $7 D$. As observed for sEPSCs, 

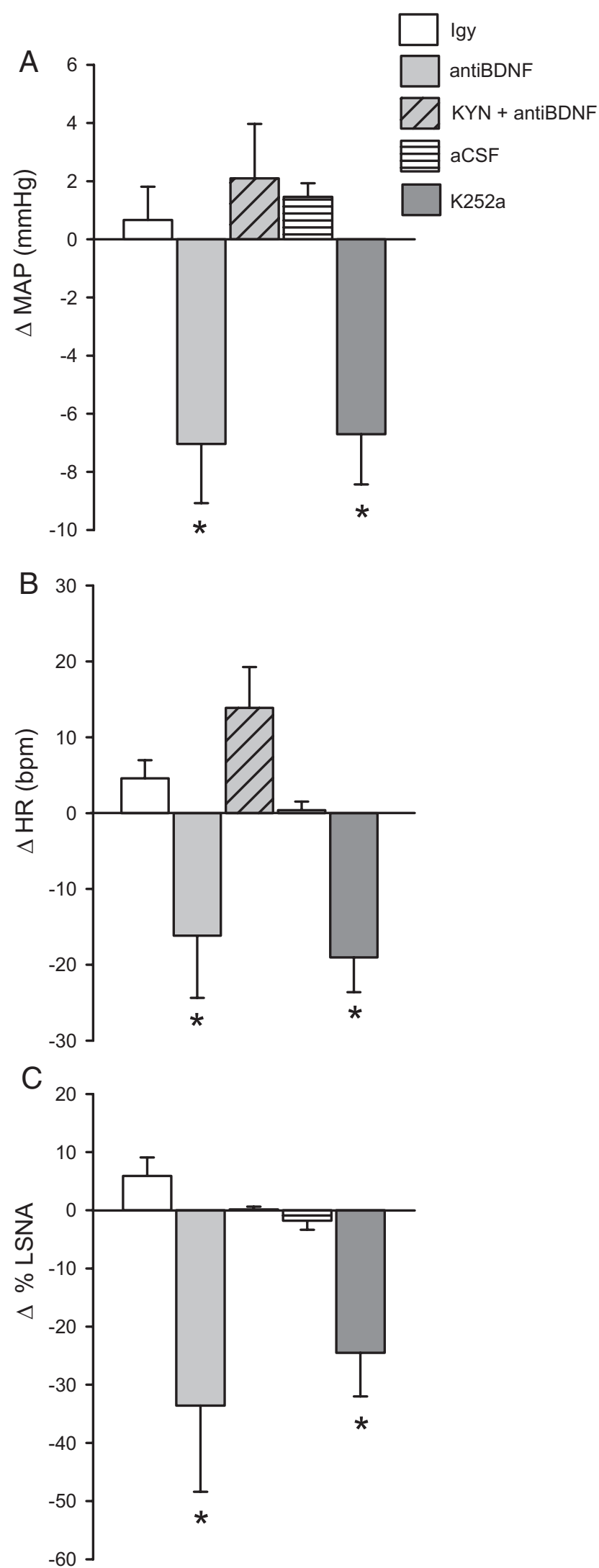

Figure 5. Blockade of either endogenous BDNF or TrkB signaling in the nTS decreases ABP, $H R$, and LSNA. A-C, The effects of blocking endogenous BDNF with bilateral microinjection of anti-BDNF (Light gray bar; $10 \mu \mathrm{g} / \mathrm{ml} ; 90 \mathrm{nl} ; n=5$ ) or a tyrosine kinase inhibitor (Dark gray bar; K252a; $200 \mathrm{~nm} ; 90 \mathrm{nl} ; n=4)$ on MAP $(\boldsymbol{A})$, HR (B), and LSNA (C) were evaluated. Anti-BDNF and K252a reduced MAP, HR, and LSNA by a similar magnitude. Prior microinjection of KYN $(40 \mathrm{~mm}$,
A

Control

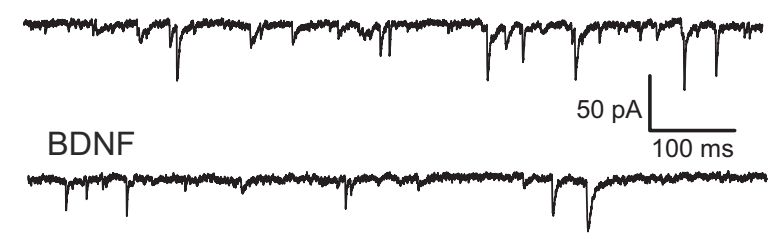

B

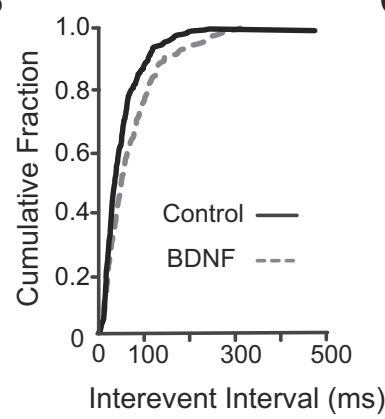

C

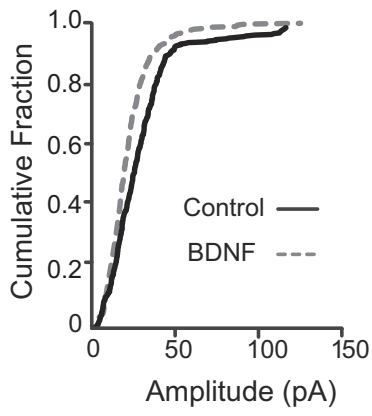

D
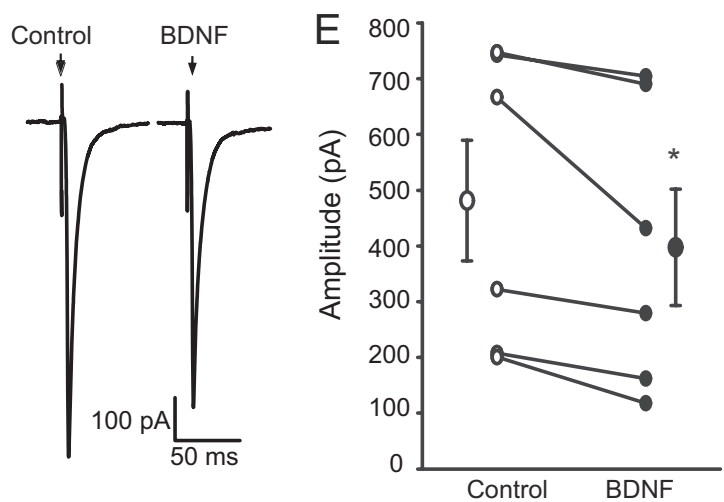

Figure 6. Exogenous BDNF decreases EPSCs in the nTS slice preparation. $\boldsymbol{A}$, Representative current tracing from an individual nTS cell illustrating sEPSCs under control conditions (upper trace) and in the presence of BDNF (lower trace). Note the decrease in sEPSC frequency and amplitude with BDNF. $B$, Cumulative probability plots of sEPSC interevent intervals $(n=6,10$ $\mathrm{ms}$ bin) under control conditions and in the presence of exogenous BDNF, showing a significant rightward shift (decreased sEPSC frequency) induced by $\operatorname{BDNF}(p<0.05$, Kolmogorov-Smirnov test). C, Cumulative probability plots of sEPSC amplitude ( $n=6,2 \mathrm{pA}$ bin) under control conditions and in the presence of BDNF, showing a significant leftward shift (reduced sEPSC amplitude) induced by BDNF ( $p<0.05$, Kolmogorov-Smirnov test). D, Representative TSEPSCS (average of 20 traces) under control conditions (aCSF, left trace) and in the presence of BDNF (right trace). Bath application of BDNF reduced the amplitude of TS-evoked EPSCs at 0.5 $\mathrm{Hz}$. Cells were voltage-clamped at $-60 \mathrm{mV}$. Arrows indicate stimulus artifact. $E$, Comparison of TS-EPSC amplitude ( $n=6$ ) in controls (open bar) and during BDNF (black bar) application. Small, connected symbols indicate data from individual neurons. Larger symbols indicate mean data. Note the reduction in amplitude in the presence of $\operatorname{BDNF}\left({ }^{*} p<0.05\right.$, paired $t$ test).

K252a increased the amplitude of TS-EPSCs. Under control conditions mean EPSC amplitude averaged $378.3 \pm 114.0$ pA. Bath application of K252a significantly increased EPSC amplitude by $19.8 \pm 6.5 \%$. to $466.2 \pm 141.7 \mathrm{pA}$ (Fig. $7 E$ ). Increases in evoked EPSC amplitude with K252a were accompanied by an increase in input resistance (control: $574.3 \pm 115.4$ vs K252a: $991.5 \pm 237.4$

$\leftarrow$

$60 \mathrm{nl} ; n=4$ ) into the nTS blocked the depressor, bradycardic and sympathoinhibitory response to anti-BDNF (Light gray, diagonal stripe bar). Controls IgY (white bar) and aCSF (white, horizontal stripe bar) had no significant effect on MAP, HR, or LSNA. Responses to IgY, aCSF and anti-BDNF in the presence of KYN were not significantly different from each other. ${ }^{*} p<0.05$ vs baseline. 


\section{A Control}

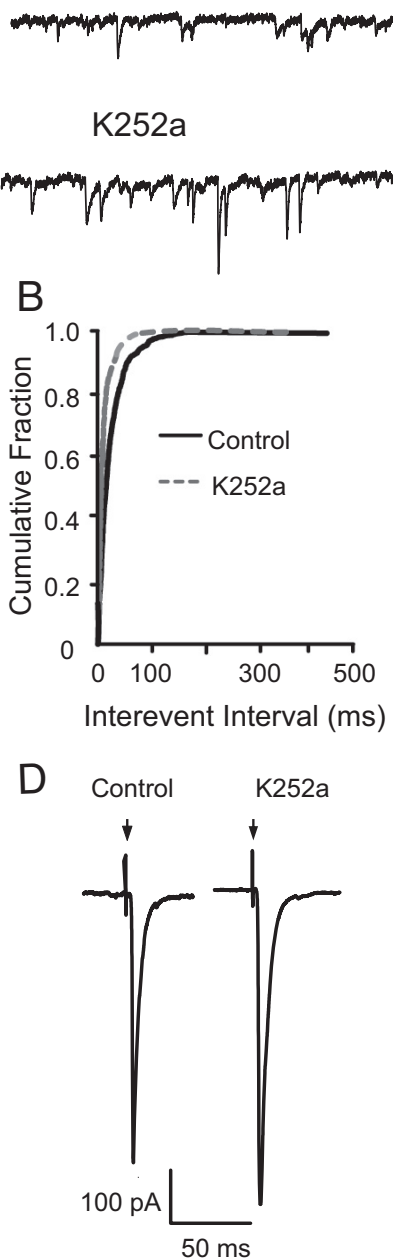

A

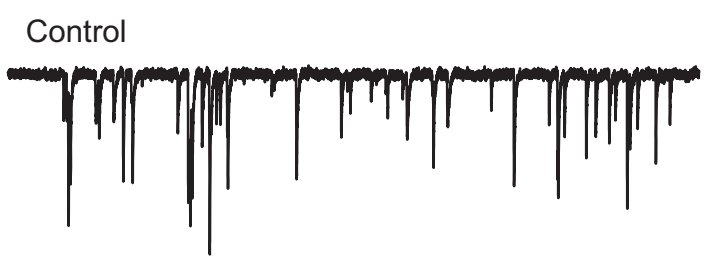

BDNF

C
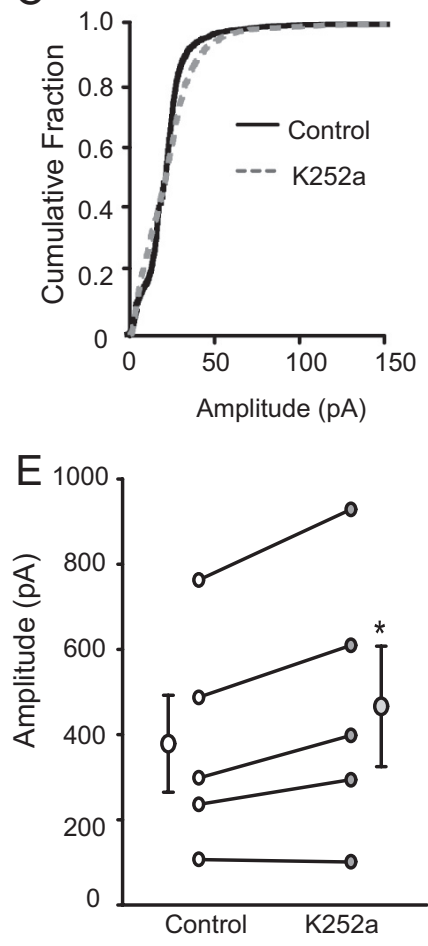

Figure 7. Blockade of TrKB receptors with K252a increases EPSCS. A, Representative current tracing of sEPSCs from an individual cell under control conditions (upper trace) and during bath application of K252a (lower trace). Note the increase in frequency and amplitude of events in the presence of K252a. B, Cumulative probability plot of sEPSC interevent interval $(n=6,10 \mathrm{~ms}$ bin) illustrates a significant leftward shift in the presence of K252a ( $p<0.05$, KolmogorovSmirnov test), indicating increased SEPSC frequency compared with control. C, Cumulative probability plot of sEPSC amplitude ( $n=6,2 \mathrm{pA}$ bin) illustrates a significant rightward shift in the presence of K252a $(p<0.05$, Kolmogorov-Smirnov), indicating increased SEPSC amplitude compared with control. $\boldsymbol{D}$, Representative tracings (average of 20 traces) of TS-evoked EPSCS elicited at $0.5 \mathrm{~Hz}$ during control (aCSF, left) and K252a (right). Blockade of tyrosine kinase receptors with K252a potentiated the amplitude of TS-EPSCS. The cell was voltage-clamped at $-60 \mathrm{mV}$. Arrows indicate stimulus artifact. $E$, Comparison of TS-EPSC amplitude under control conditions (open bar) and during superfusion of K252a (Gray bar) $(n=5)$. Small, connected symbols indicate data from individual neurons. Larger symbols indicate mean data. K252a increased the amplitude of TS-evoked EPSCS $\left({ }^{*} p<0.05\right.$, paired $t$ test).

$\mathrm{M} \Omega$ ). Additionally, K252a resulted in a net inward holding current (Control: $-13.3 \pm 11.9$ vs BDNF: $-29.6 \pm 9.7 \mathrm{pA}$ ).

BDNF attenuates miniature EPSC amplitude

To evaluate the potential contribution of presynaptic versus postsynaptic actions of BDNF on synaptic transmission, miniature (m)EPSCs were examined. Miniature currents, recorded in the presence of TTX and gabazine, represent the quantal release of glutamate, independent of action potential activity. As illustrated by the example shown in Figure $8 A$, BDNF significantly reduced the amplitude of mEPSCs (control, $27.2 \pm 3.5 \mathrm{pA}$ vs BDNF, $22.2 \pm 2.7 \mathrm{pA}$, an $18 \pm 6 \%$ reduction, $n=8, p<0.05$ ) but had no

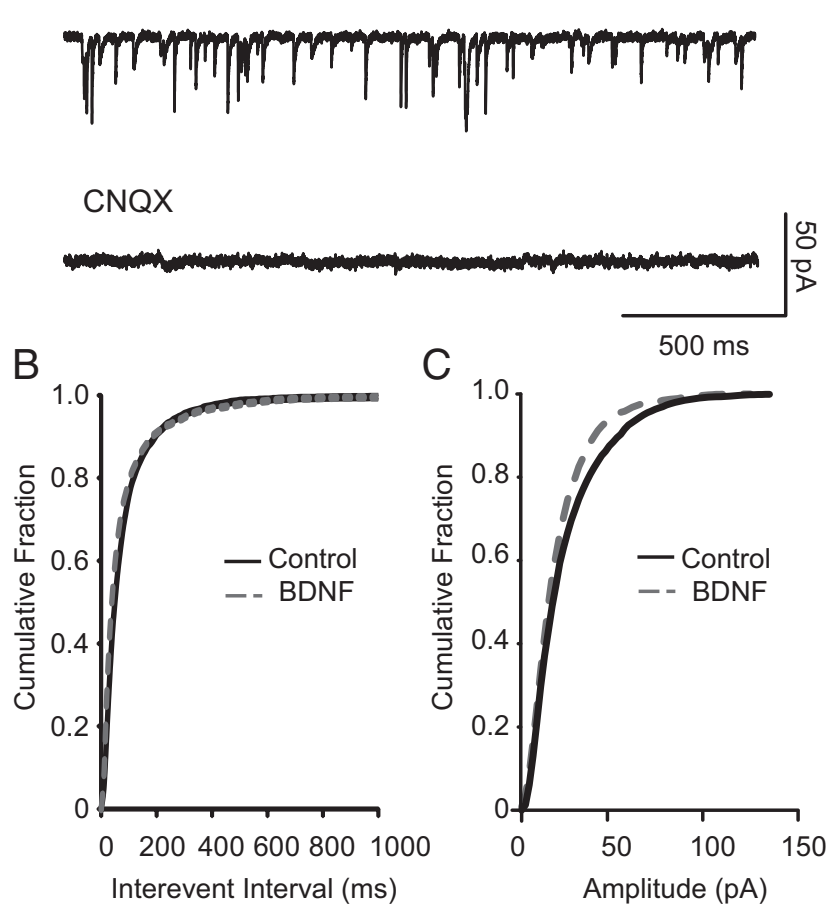

Figure 8. BDNF decreases mEPSC amplitude without affecting frequency. $\boldsymbol{A}$, Representative example of mEPSCs during control (top), BDNF (100 ng/ml, middle) and CNQX (10 $\mu \mathrm{m}$, bottom). All recordings were made with gabazine $(25 \mu \mathrm{M})$ and Tetrodotoxin $(1 \mu \mathrm{M})$ present in the bath. Note the decrease in current amplitude but not frequency with bath application of BDNF. $\boldsymbol{B}$, The cumulative probability plot of $\mathrm{mEPSC}$ interevent intervals $(10 \mathrm{~ms}$ bin) revealed no change in the curve due to BDNF. C, The cumulative probability plot of mEPSC amplitudes ( $2 \mathrm{pA}$ bin) revealed a significant leftward shift in the presence of $\operatorname{BDNF}(p<0.05$, Kolmogorov-Smirnov test).

effect on mEPSC frequency (control, $11.1 \pm 3.7 \mathrm{~Hz}$ vs BDNF, $11.4 \pm 3.5 \mathrm{~Hz}, p>0.05, n=8$ ). The distributions of mEPSC interevent interval and amplitude are shown in Figure 8, $B$ and $C$. Moreover, mEPSCs were reversibly blocked by CNQX. Together, these data indicate that BDNF most likely decreases glutamatergic synaptic transmission through a postsynaptic mechanism.

\section{$B D N F$ attenuates current-evoked APD}

The effects of BDNF on postsynaptic properties were further evaluated in a subset of animals by recording action potentials in current-clamp mode at resting membrane potential. Bath application of BDNF did not change membrane potential (Control: $-61.3 \pm 2.8$ vs BDNF: $-58.7 \pm 3.5 \mathrm{mV}, n=6$, paired $t$ test: $p=$ 0.09 ) but significantly decreased input resistance (Control: $649.7 \pm 127.5$ vs BDNF: $358.9 \pm 95.6 \mathrm{M} \Omega$ ). Microinjection of depolarizing current (500 ms, 10 pA steps) induced APD in both the absence and presence of BDNF, and initial action potential properties were not significantly different between the two conditions (Control vs BDNF: Amplitude: $63.9 \pm 4.0$ vs $62.8 \pm 4.2$ $\mathrm{mV}$; threshold: $-39.4 \pm 5.4$ vs $-35.4 \pm 1.9 \mathrm{mV}$; half-width: $0.9 \pm 0.1$ vs $1.1 \pm 0.1 \mathrm{~ms}$; rise time: $0.5 \pm 0.1 \mathrm{vs} 0.5 \pm 0.04 \mathrm{~ms}$; decay time: $1.0 \pm 0.2$ vs $0.9 \pm 0.1 \mathrm{~ms}$ ). As illustrated in Figure $9 A$, progressively increasing the depolarizing current augmented dis- 
A Control BDNF
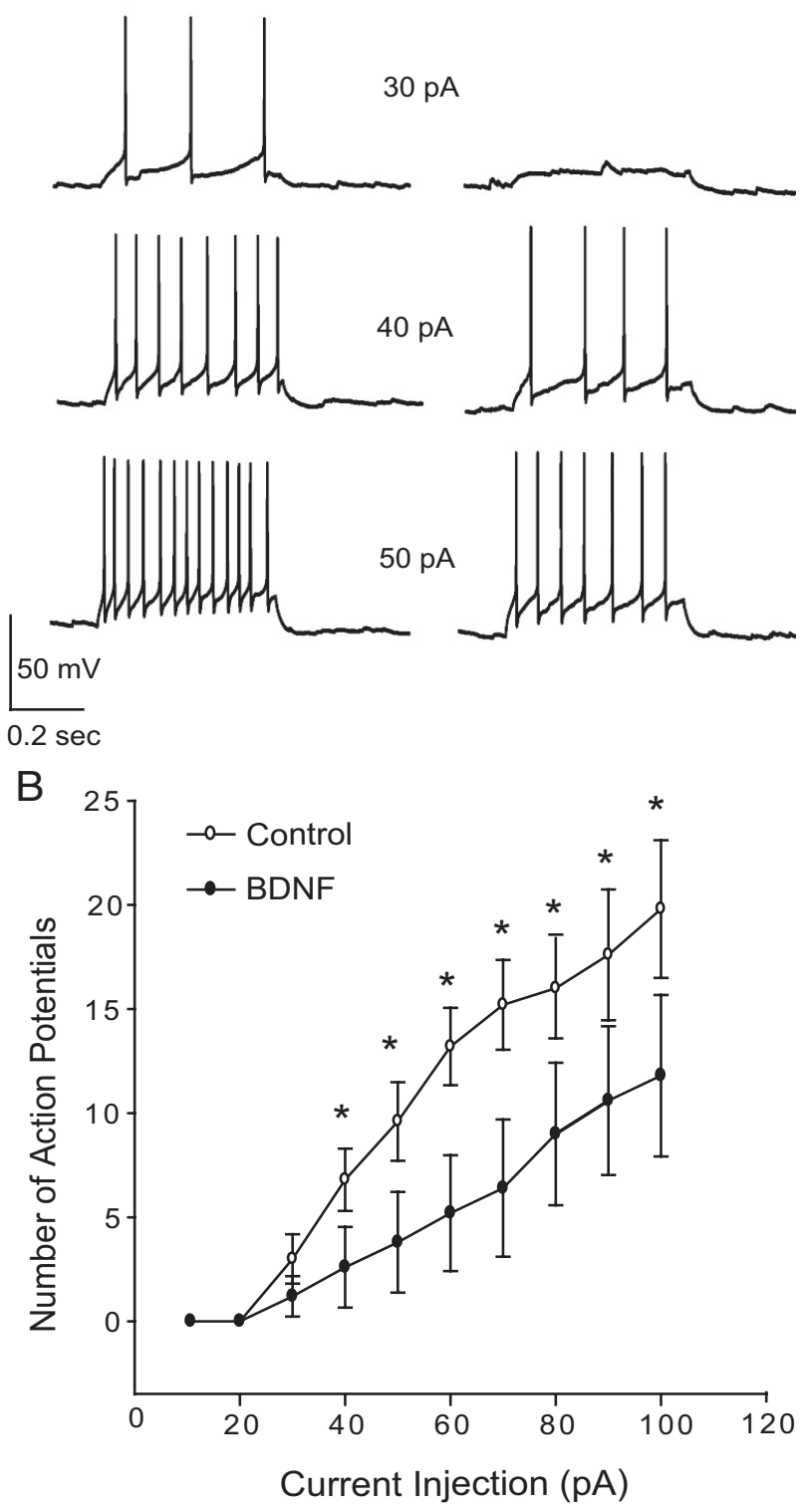

Figure 9. BDNF decreases nTS action potential discharge. $\boldsymbol{A}$, Representative traces of neuronal discharge under control conditions (left) and in the presence of $B D N F$ (right) in response to 30,40 , and $50 \mathrm{pA}(500 \mathrm{~ms})$ current injection steps $(n=6)$ in an individual nTS cell. Note that increasing depolarizing current injection increased action potential discharge. However, APD was less following application of BDNF. $B$, Group comparison of APD of cells at resting membrane potential in response to current injection steps ranging from $10-100 \mathrm{pA}$. Note the decreased APD in the presence of BDNF at currents $\geq 40 \mathrm{pA}\left({ }^{*} p<0.05\right.$ vs control, two-way repeated-measures ANOVA).

charge under control conditions and in the presence of BDNF. However, in the same neuron BDNF reduced the number of action potentials at a given current compared with control. Plotting the number of action potentials induced by increasing depolarizing current demonstrates that BDNF significantly reduced the number of action potentials generated at currents $\geq 40 \mathrm{pA}$ (Fig. 9B).

\section{Discussion}

Our data demonstrate that endogenous BDNF tonically regulates blood pressure, heart rate and sympathetic nervous system activity in vivo and modulates glutamatergic excitatory synaptic transmission in nTS. In particular, we found that scavenging endogenous BDNF by microinjection of function-blocking BDNF antibodies into the medial nTS, a region critical for reflex modulation of sympathetic outflow, produced depressor responses, bradycardia and sympathoinhibition. Analysis of nTS synaptic function in vitro revealed that BDNF acts primarily at postsynaptic sites to inhibit excitatory transmission at glutamatergic primary afferent synapses.

A role for BDNF in regulation of cardiovascular pathways in nTS is consistent with the abundant expression of BDNF protein in presynaptic and postsynaptic elements in nTS circuits (Conner et al., 1997). Most notably, BDNF is expressed at high levels by vagal and glossopharyngeal afferents (Brady et al., 1999), including baroafferents (Martin et al., 2008), that provide presynaptic input to visceral sensory relay neurons in nTS. Moreover, the BDNF receptor, TrkB, is expressed by second-order nTS neurons, in close apposition to BDNF-positive presynaptic boutons (Kline et al., 2010). In addition, BDNF is released from vagal afferent neurons in response to patterned electrical stimulation (Balkowiec and Katz, 2000), including at frequencies associated with baroreceptor activation (Andresen and Kunze, 1994). Finally, previous studies of isolated nTS neurons demonstrated that exogenous BDNF can potently modulate excitatory postsynaptic responses to applied glutamate in vitro by inhibiting AMPA receptor-mediated currents (Balkowiec et al., 2000). The present findings, however, are the first demonstration that endogenous BDNF modulates synaptic transmission and autonomic function in nTS.

Our studies focused on the role of BDNF in the medial nTS, a critical site for baroreceptor modulation of sympathetic outflow. Normally, baroreceptor afferents provide glutamatergic excitatory drive to mnTS neurons which, in turn, activate inhibitory interneurons in the caudal ventrolateral medulla (CVLM) to reduce sympathetic drive from the rostral VLM (RVLM) in response to increases in mean arterial pressure. This homeostatic circuit is tonically active and modulates sympathetic nervous system activity even at baseline levels of MAP. One possibility, therefore, is that the pressor and sympathoexcitatory effects of exogenous BDNF result from inhibition of mnTS neurons in the baroreflex arc. This possibility is supported by the fact that immunoneutralization of endogenous BDNF in mnTS results in a depressor response and sympathoinhibition. Interestingly, the majority of CVLM-projecting nTS neurons are second-order neurons (Bailey et al., 2006) which, as we have previously shown, express TrkB (Balkowiec et al., 2000; Kline et al., 2010). Thus, BDNF may enhance sympathoexcitatory drive by inhibiting primary afferent transmission through the baroreflex pathway. This possibility is further supported by our in vitro experiments demonstrating that exogenous BDNF inhibits postsynaptic responses of mnTS neurons to primary afferent stimulation. Such a mechanism does not exclude other possibilities as well, including a role for BDNF in modulation of interneurons that regulate synaptic strength in the baroreflex pathway in nTS (Andresen and Kunze, 1994).

To define potential mechanisms by which BDNF modulates synaptic transmission in mnTS, we characterized electrophysiological properties of second-order nTS neurons in the absence and presence of exogenous BDNF in brainstem slices in vitro. BDNF decreased the amplitude of spontaneous, evoked and miniature EPSCs, as well as TS-evoked APD but did not change mEPSC frequency, paired-pulse ratio or failure rate. Together, these data are consistent with postsynaptic modulation of synap- 
tic transmission by BDNF. They also extend previous studies in which BDNF attenuated AMPA-mediated currents in isolated second-order nTS neurons (Balkowiec et al., 2000). BDNF also attenuated spiking induced by injecting depolarizing currents into the postsynaptic cell, decreased input resistance and increased outward holding current. These data suggest that BDNF exerts additional postsynaptic effect(s) independent of synaptic transmission.

We also found evidence for potential presynaptic effects of BDNF on mnTS circuit function. BDNF decreased the frequency of sEPSCs, an indication of reduced spontaneous neurotransmitter release from presynaptic neurons. These currents represent network activity arising from multiple sources, including sensory afferents, central inputs and interneurons. A presynaptic effect of BDNF on sensory afferents is unlikely, given that TrkB is not detected in vagal afferents (Zhou and Helke, 1996) or primary afferent terminals in nTS after birth (Kline et al., 2010). Moreover, BDNF produced no change in the frequency of mEPSCs, paired-pulse ratio or EPSC failure rate following afferent stimulation, parameters that are sensitive to presynaptic changes in quantal neurotransmitter release. Thus, decreased sEPSC frequency in response to exogenous BDNF is likely not due to effects directly at presynaptic terminals, but may reflect reduced transmitter release due to decreased spiking in cells other than primary afferents, including local interneurons, onto second-order mnTS neurons.

Our results suggest BDNF blunts excitatory drive in the mnTS. However, GABA, the major inhibitory transmitter in nTS, contributes to cardiorespiratory regulation in nTS (Andresen and Kunze, 1994; Zhang and Mifflin, 2010) and BDNF may enhance GABAergic signaling (Jovanovic et al., 2004). GABAergic transmission does not influence EPSCs in nTS of Mecp2-null mice that exhibit reduced BDNF levels and exaggerated excitatory synaptic signaling (Kline et al., 2010). Furthermore we recorded EPSCs near the reversal potential for chloride where the influence of GABAergic signaling is minimal, and the effect of BDNF to reduce TS-EPSC amplitude was maintained in the presence of the $\mathrm{GABA}_{\mathrm{A}}$ receptor antagonist gabazine. Our data suggest that glutamatergic neurotransmission in particular is a major target of BDNF actions in mnTS. BDNF inhibited neurotransmission between visceral afferents and second order nTS neurons, a synapse that requires glutamatergic signaling (Andresen and Yang, 1990; Kline et al., 2002). In addition, TS-EPSCs and mEPSCs that were modulated by BDNF were blocked by the non-NMDA receptor antagonist CNQX. In vivo, cardiovascular responses to immunoneutralization of endogenous BDNF were eliminated by iGluR blockade with KYN. It is possible that KYN may have eliminated the ability of nTS neurons to respond to any subsequent manipulations, including anti-BDNF treatment. However, previous studies have shown that while KYN abolishes responses to sensory afferent stimulation it does not abolish nTS responses to other excitatory agonists (Pawloski-Dahm and Gordon, 1992; Foley et al., 1998; Mueller and Hasser, 2006). Thus, while an effect on GABAergic transmission cannot be completely ruled out, our data indicate that BDNF modulates glutamatergic function in nTS in vitro and in vivo. Our in vitro data indicate that BDNF acts, at least in part, at synapses between primary visceral afferents and second order nTS neurons (present study and Kline et al., 2010), although additional sites of action may also play a role in vivo.

Modulation of baroreflex function is critical to maintaining cardiovascular homeostasis under physiological and pathological conditions (Eckberg et al., 1971; La Rovere et al., 1998). A sym- pathoexcitatory effect of BDNF in nTS is consistent with BDNF tonically blunting baroreflex-mediated sympathoinhibition. Because BDNF release is activity-dependent (Balkowiec and Katz, 2000), its effects may be greatest at elevated pressure when baroafferent activity is highest. Increased BDNF release may therefore also contribute to short-term plasticity of the reflex such as occurs with acute baroreflex resetting in response to increased afferent activity.

BDNF may also play a role in long-term changes in reflex function such as chronic baroreflex resetting in hypertension. Given that DOCA-salt hypertensive rats exhibit increased BDNF mRNA in the NPG (Jenkins et al., 2007), our data raise the possibility that increased BDNF/TrkB signaling in the nTS baroreflex pathway may contribute to the pathophysiology of chronic hypertension by blunting sympathoinhibition.

In light of the fact that BDNF levels in nTS can influence synaptic transmission and autonomic outflow, nTS may be a critical locus at which derangements in BDNF signaling contribute to developmental disorders of autonomic control. For example, RTT patients, as well as mouse models of the disease (Mecp2 loss of function mutants), exhibit decreased BDNF expression and severe autonomic dysfunction (Weese-Mayer et al., 2006; Katz et al., 2009). In Mecp2-null mice, BDNF expression is reduced in areas critical for autonomic control including the nodose ganglia and nTS (Wang et al., 2006b). Moreover, secondorder nTS neurons in Mecp2-null mice exhibit increased EPSC amplitude and AP firing compared with wild types, which are rescued by exogenous BDNF (Kline et al., 2010). These results are consistent with a role for BDNF in inhibiting excitatory synaptic transmission in nTS, as described in the present study. The net result of reduced $\mathrm{BDNF}$ expression on autonomic dysfunction in RTT is likely to be complex, as diverse brainstem cell groups involved in autonomic control exhibit BDNF deficits in RTT mice (Kline et al., 2010). Thus, although the present findings alone cannot explain autonomic phenotypes in RTT, these data support the concept that decreased BDNF availability in the nTS may contribute to autonomic dysregulation in RTT and possibly other diseases as well (Katz et al., 2009; Kline et al., 2010).

In summary, our data demonstrate a previously unrecognized role for BDNF in mnTS in the regulation of cardiovascular homeostasis. BDNF contributes to ongoing regulation of blood pressure and sympathetic activity due, at least in part, to modulation of glutamatergic neurotransmission in mnTS. These findings raise the possibility that BDNF signaling in nTS may be a therapeutic target for regulating cardiovascular homeostasis in the setting of disorders of autonomic control.

\section{References}

Alonso M, Vianna M, Izquierdo I, Medina J (2002) Signaling mechanisms mediating BDNF modulation of memory formation in vivo in the hippocampus. Cell Mol Neurobiol 22:663-674.

Andresen MC, Kunze DL (1994) Nucleus tractus solitarius-gateway to neural circulatory control. Annu Rev Physiol 56:93-116.

Andresen MC, Yang M (1990) Non-NMDA receptors mediate sensory afferent synaptic transmission in medial nucleus tractus solitarius. Am J Physiol 259:H1307-H1311.

Aylwin ML, Horowitz JM, Bonham AC (1998) Non-NMDA and NMDA receptors in the synaptic pathway between area postrema and nucleus tractus solitarius. Am J Physiol 275:H1236-H1246.

Bailey T, Hermes S, Andresen M, Aicher S (2006) Cranial visceral afferent pathways through the nucleus of the solitary tract to caudal ventrolateral medulla or paraventricular hypothalamus: target-specific synaptic reliability and convergence patterns. J Neurosci 26:11893-11902.

Balkowiec A, Katz DM (2000) Activity-dependent release of endogenous 
brain-derived neurotrophic factor from primary sensory neurons detected by ELISA in situ. J Neurosci 22:10399-10407.

Balkowiec A, Kunze DL, Katz DM (2000) Brain-derived neurotrophic fact acutely inhibits AMPA-mediated currents in developing sensory relay neurons. J Neurosci 20:1904-1911.

Brady R, Zaidi S, Mayer C, Katz DM (1999) BDNF is a target-derived survival factor for arterial baroreceptor and chemoafferent primary sensory neurons. J Neurosci 19:2131-2142.

Conner JM, Lauterborn JC, Yan Q, Gall CM, Varon S (1997) Distribution of brain-derived neurotrophic factor (BDNF) protein and mRNA in the normal adult rat CNS: evidence of anterograde axonal transport. J Neurosci 17:2295-2313.

Doyle MW, Andresen MC (2001) Reliability of monosynaptic sensory transmission in brain stem neurons in vitro. J Neurophysiol 85:2213-2223.

Eckberg DL, Drabinsky M, Braunwald E (1971) Defective cardiac parasympathetic control in patients with heart disease. New Engl J Med 877-883.

Faraguna U, Vyazovkiy W, Nelson A, Tononi G, Cirelli C (2008) A causal role for brain-derived neurotrophic factor in the homeostatic regulation of sleep. J Neurosci 28:4088-4095.

Foley CM, Moffitt JA, Hay M, Hasser EM (1998) Glutamate in the nucleus of the solitary tract activates both ionotropic and metabotropic glutamate receptors. Am J Physiol 275:R1858-R1866.

Gordon FJ, Leone C (1989) Is L-glutamate a neurotransmitter of baroreceptor information in the nucleus of the tractus solitarius? J Pharmacol Exp Ther 250:953-962.

Husson I, Rangon C, Lelievre V, Bemelmans A, Sachs P, Mallet J, Kosofsky B, Gressens P (2005) BDNF-induced white matter neuroprotection and stage-dependent neuronal survival following a neonatal excitotoxic challenge. Cereb Cortex 15:250-261.

Jenkins V, Pricher M, O’Donaughty T, Hsieh HY, Brooks V, Balkowiec A (2007) Activity-dependent regulation of BDNF expression in nodose ganglia of DOCA-salt hypertensive rats. Soc Neurosci Abstr 33:139.11.

Jovanovic JN, Thomas P, Kittler JT, Smart TG, Moss SJ (2004) Brainderived neurotrophic factor modulates fast synaptic inhibition by regulating $\mathrm{GABA}(\mathrm{A})$ receptor phosphorylation, activity, and cell-surface stability. J Neurosci 24:522-530.

Kang H, Schuman EM (1995) Long-lasting neurotrophin-induced enhancement of synaptic transmission in the adult hippocampus. Science 267:1658-1662.

Katz D, Dutschmann M, Ramirez J, Hilaire G (2009) Breathing disorders in Rett Syndrome: progressive neurochemical dysfunction in the respiratory network after birth. Respir Physiol Neurobiol 168:101-108.

Kline DD, Takacs KN, Ficker E, Kunze DL (2002) Dopamine modulates synaptic transmission in the nucleus of the solitary tract. J Neurophysiol $88: 2736-2744$.
Kline DD, Kunze DL, Katz DM (2010) Exogenous brain-derived neurotrophic factor rescues synaptic dysfunction in Mecp2-null mice. J Neurosci 30:5303-5310.

La Rovere MT, Bigger JT, Marcus FI, Mortara A, Schwartz PJ (1998) Baroreflex sensitivity and heart-rate variability in prediction of total cardiac mortality after myocardial infarction. Lancet 351:478-484.

Martin J, Jenkins V, Hsieh H, Balkowiec A (2008) Brain-derived neurotrophic factor in arterial baroreceptor pathways: implications for activity-dependent plasticity at baroreceptor synapses. J Neurochem 108:450-464.

Mueller PJ, Hasser EM (2006) Putative role of the NTS in alterations in neural control of the circulation following exercise training in rats. Am J Physio Regul Integr Comp Physiol 290:R383-R392.

Ogier M, Wang H, Hong E, Wang Q, Greenberg M, Katz D (2007) Brainderived neurotrophic factor expression and respiratory function improve after ampakine treatment in a mouse model of Rett syndrome. J Neurosci 27:10912-10917.

Ohta H, Talman WT (1994) Both NMDA and non-NMDA receptors in the NTS participate in the baroreceptor reflex in rats. Am J Physiol 267:R1065-R1070.

Pawloski-Dahm C, Gordon FJ (1992) Evidence for a kynurenateinsensitive glutamate receptor in nucleus tractus solitarii. Am J Physiol 262:H1611-1615.

Paxinos G, Watson C (1998) The rat brain in stereotaxic coordinates. New York: Elsevier.

Sanchez A, Matthews B, Meynard M, Hu B, Javed S, Cohen Cory S (2006) BDNF increases synapse density in dendrites of developing tectal neurons in vivo. Development 133:2477-2486.

Wang H, Ward N, Boswell M, Katz DM (2006a) Secretion of brain-derived neurotrophic factor from brain microvascular endothelial cells. Eur J Neurosci 23:1665-1670.

Wang H, Chan SA, Ogier M, Hellard D, Wang Q, Smith C, Katz DM (2006b) Dysregulation of brain-derived neurotrophic factor expression and neurosecretory function in Mecp2-null mice. J Neurosci 26:10911-10915.

Weese-Mayer DE, Lieske SP, Boothby CM, Kenny AS, Bennett HL, Silverstri JM, Ramirez JM (2006) Autonomic nervous system dysregulation: breathing and heart rate perturbation during wakefulness in young girls with Rett syndrome. Pediatr Res 4:443-449.

Zhang W, Mifflin S (2010) Plasticity of GABAergic mechanisms within the nucleus of the solitary tract in hypertension. Hypertension 201-206.

Zhou H, Helke C (1996) Presence and localization of neurotrophin receptor tyrosine kinase (TrkA, TrkB, TrkC) mRNAs in visceral afferent neurons of the nodose and petrosal ganglia. Brain Res Mol Brain Res 38:63-70. 